\title{
Substructure of the galaxy clusters in the REXCESS sample: observed statistics and comparison to numerical simulations
}

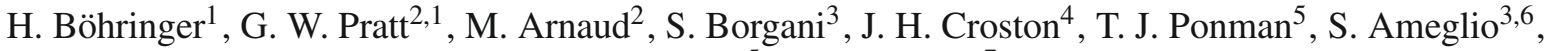 \\ R. F. Temple ${ }^{5}$, and K. Dolag ${ }^{7}$
}

1 Max-Planck-Institut für extraterrestrische Physik, 85748 Garching, Germany

e-mail: hxb@mpe.mpg.de

2 Laboratoire AIM, IRFU/Service d'Astrophysique - CEA/DSM - CNRS - Université Paris Diderot, Bât. 709, CEA-Saclay, 91191 Gif-sur-Yvette Cedex, France

3 Dipartimento di Astronomia dell’Universitá di Trieste, via Tiepolo 11, 34133 Trieste, Italy

4 School of Physics and Astronomy, University of Southampton, Southampton, SO17 1BJ, UK

5 School of Physics and Astronomy, University of Birmingham, Edgbaston, Birmingham B15 2TT, UK

${ }^{6}$ University of Southern California, Los Angeles, CA, USA

7 Max-Planck-Institut für Astrophysik, 85748 Garching, Germany

Received 18 December 2009 / Accepted 3 February 2010

\section{ABSTRACT}

\begin{abstract}
We study the substructure statistics of a representative sample of galaxy clusters by means of two currently popular substructure characterisation methods, power ratios and centroid shifts. We use the 31 clusters from the REXCESS sample, compiled from the southern ROSAT All-Sky cluster survey (REFLEX) with a morphologically unbiased selection in X-ray luminosity and redshift, all of which have been reobserved with XMM-Newton. The main goals of this work are to study the relationship between cluster morphology and other bulk properties, and the comparison of the morphology statistics between observations and numerical simulations. We investigate the uncertainties of the substructure parameters via newly-developed Monte Carlo methods, and examine the dependence of the results on projection effects (via the viewing angle of simulated clusters), finding that the uncertainties of the parameters can be quite substantial. Thus while the quantification of the dynamical state of individual clusters with these parameters should be treated with extreme caution, these substructure measures provide powerful statistical tools to characterise trends of properties in large cluster samples. The centre shift parameter, $w$, is found to be more sensitive in general and offers a larger dynamic range than the power ratios. For the REXCESS sample neither the occurence of substructure nor the presence of cool cores depends on cluster mass; however a weak correlation with X-ray luminosity is present, which is interpreted as selection effect. There is a significant anti-correlation between the existence of substantial substructure and cool cores. The simulated clusters show on average larger substructure parameters than the observed clusters, a trend that is traced to the fact that cool regions are more pronounced in the simulated clusters, leading to stronger substructure measures in merging clusters and clusters with offset cores. Moreover, the frequency of cool regions is higher in the simulations than in the observations, implying that the description of the physical processes shaping cluster formation in the simulations requires further improvement.
\end{abstract}

Key words. X-rays: galaxies: clusters - galaxies: clusters: intracluster medium - cosmology: observations

\section{Introduction}

There are two major far-reaching interests that motivate our understanding the population of galaxy clusters. Firstly, they are ideal test objects to check the likelihood of a given cosmological model to describe our Universe; secondly, they are very important probes of the astrophysical and chemical evolution of the baryonic component of the Universe (e.g. Rosati et al. 2002; Schuecker et al. 2003a,b; Voit 2005; Vikhlinin et al. 2003, 2009; Rozo et al. 2007; Henry et al. 2009; Mantz et al. 2008). A good understanding and observational characterisation of galaxy clusters is required to attain these goals.

X-ray observations provide an essential window into the study of galaxy clusters, as the presence of X-ray radiation implies and traces a well developed gravitational well (e.g. Sarazin 1986; Böhringer 2008), offering the best starting point for the characterisation of the cluster mass and dynamical state. The temperature of the hot intracluster medium is related to the depth of the potential well, and its distribution is related to the dynamical state of the system. In this study we use statistical measures of substructure observed in the X-ray images, which provide a projected view of the ICM structure, to obtain an impression of the cluster's dynamical state ${ }^{1}$. Ideally one would like to base such a study on a known relation between a substructure parameterization and a measure of the deviation from dynamical equilibrium of the cluster. While this could be an important aim for a future study based on simulations ${ }^{2}$, we pursue here the more qualitative goal of using substructure measures without a calibrated relation to a physical quantity to sort clusters into a relative ranking order.

The two direct applications of substructure measures are (i) the study of the influence of substructure on cluster scaling

\footnotetext{
${ }^{1}$ Galaxy velocity distributions would provide complementary information on the cluster substructure along the line-of-sight (e.g. Girardi et al. 1997; Biviano et al. 2006).

${ }^{2}$ Indeed, Yang et al. (2008) have recently made a first approach in this direction by comparing substructure measures of simulated clusters with the time since the last merger.
} 
relations, e.g., of global X-ray properties, and (ii) a comparison of the distribution of the substructure measures in statistically representative samples of observed and simulated galaxy clusters $^{3}$. The latter is an important test of how well simulated cluster morphologies correspond to the observed cluster population. Any difference will most probably point to a shortcoming in the description of important physical processes in the simulations. If we want the simulations to correspond well enough to the real world, so that we can draw essential conclusions, we have to perform a series of tests to compare the details of the cluster appearance in simulated and observed objects. While previous studies focussed on reproducing the scaling relations of global cluster parameters (e.g. Borgani et al. 2004; Voit et al. 2003; McCarthy et al. 2004, 2008; Poole et al. 2006, 2007, 2008; Kay et al. 2007; Borgani \& Kravtsov 2009), ours is one of the first such tests to use morphology.

In the past, a series of different methods have been used to characterise substructure in X-ray images of galaxy clusters. Optical substructure has been characterised in various ways by e.g. Fitchett \& Webster (1987), Beers et al. (1992) and Bird \& Beers (1993). Several of these methods were tested and summarised by Pinkey et al. (1996), along with methods that can be applied in a similar way to photon distributions. Schuecker et al. (2001) used the methods of Pinkey et al. to obtain substructure statistics in X-ray detected galaxy clusters from the ROSAT All-Sky Survey, and tested the correlation of the substructure measures with the occurrence of radio halos and with the object's location in the large-scale structure environment. Slezak et al. (1994) applied a wavelet technique to X-ray cluster images. Gomez et al. (1997, using the Pinkney method), Hashimoto et al. (2007b), and Kolokotronis et al. (2001) compared the X-ray and optical appearance of galaxy clusters using ellipticities and center shifts. We concentrate here on two methods: centre shifts as a function of the aperture radius (e.g. Mohr et al. 1993, 1995; Poole et al. 2006; O'Hara et al. 2006) and the determination of so-called power ratios (e.g. Buote \& Tsai 1995, 1996; Jeltema et al. 2005, 2008; Valdarini 2006; Ventimiglia et al. 2008).

Any general characterisation of the galaxy cluster population must be statistical. For a comparison study with numerical simulations, a statistically unbiased sample of clusters is required that contains a representative distribution of cluster morphologies. We therefore base our work on REXCESS (Representative XMM-Newton Cluster Structure Survey, Böhringer et al. 2007), a sample that has been constructed with exactly this goal in mind. It is the special construction of the REXCESS sample that makes a correct comparison with numerical simulations possible, and this is probably the most innovative aspect of this paper. Previous publications on the properties of the REXCESS sample include Pratt et al. (2007, 2009), on the temperature profiles and the X-ray luminosity scaling relations, and Croston et al. (2008), on the gas density profiles. Further recent papers describing properties of the clusters in the REXCESS sample are Pratt et al. (2010), on the structure of the entropy profiles, Arnaud et al. (2009) on the pressure profiles and scaling relations involving the X-ray determined equivalent of the Sunyaev-Zeldovich Effect Comptonization parameter $Y_{\mathrm{X}}$, and a study of the characteristics of the brightest cluster galaxies by

\footnotetext{
${ }^{3}$ More than a decade ago one of the applications of determining the ratio of relaxed to unrelaxed clusters was aiming at constraining the mean matter density of the Universe by means of this parameter (e.g. Richstone et al. 1992; Evrard et al. 1993; Melott et al. 2001; Buote \& Xu 1997; Valdarnini et al. 1999). Since we have now better approaches for a much more precise determination of the cosmic matter density, this application is no more attractive.
}

Haarsma et al. (2010). All of the above papers have made use of the substructure parameters obtained by the analysis described herein.

The paper is structured as follows. Section 2 gives a brief description of REXCESS and introduces the substructure characterisation methods used. Section 3 describes the simulations used and their analysis. The results of the substructure analysis of the observations and simulations are discussed in Sect. 4. Correlations of the substructure measures with other global clusters parameters are studied in Sects. 5 and 6 provides a discussion and conclusions.

For the scaling of distance dependent parameters we use a flat concordance cosmological model with $H_{0}=$ $70 \mathrm{~km} \mathrm{~s}^{-1} \mathrm{Mpc}^{-1}$ and $\Omega_{\mathrm{m}}=0.3$.

\section{Data analysis}

\subsection{The REXCESS sample}

REXCESS was constructed with the aim of providing a statistically well defined collection of galaxy clusters, selected only by their X-ray luminosities and redshifts without any bias with respect to cluster morphology (for details see Böhringer et al. 2007). The full sample of 33 clusters is drawn from the REFLEX survey (Böhringer et al. 2001, 2004) such as to homogeneously cover the luminosity range of 0.4 to $20 \times 10^{44} h_{50}^{-1} \mathrm{erg} \mathrm{s}^{-1}$ in the 0.1 to $2.4 \mathrm{keV}$ band, and the redshifts were chosen so that the clusters fit optimally into the field-of-view of the XMM-Newton $\mathrm{X}$-ray telescopes, with a cluster free region for background assessment. The resulting redshift range is $z=0.0564$ to 0.1832 , with redshift increasing with the luminosity and size of the clusters. The luminosity range corresponds roughly to an ICM temperature interval from about 2 to $10 \mathrm{keV}$ (Pratt et al. 2009). With a requested exposure of at least $25 \mathrm{ks}$, more than $16 \mathrm{ks}$ observation time is left for both EMOS and EPN detectors in all but four clusters after removing soft proton flare contamination.

Three of the REXCESS targets have two or more well-separated X-ray maxima: the supercluster complex Abell 901/902 (RXC J0956.4-1004), and the bimodal systems RXC J2157.4-0747 and RXC J2152.2-1942. The latter has a close neighbour detected as a well-separated region of X-ray emission in the ROSAT All-Sky Survey, but the two components overlap within their values of $R_{500}$ (defined below) and their $\mathrm{X}$-ray emission clearly overlaps in the deeper XMM-Newton images. The Abell 901/902 complex contains at least three regions of extended X-ray emission with several additional bright X-ray point sources due to AGN. Since the X-ray source regions of these systems cannot be treated by the analysis performed in this work without manual decomposition which may be subjective, we exclude these two objects from further analysis (they were also excluded in Croston et al. 2008 and Pratt et al. 2007, 2009), The third system, RXC J2157.4-0747, has two components that can be well separated. In the following analysis the centre is defined to coincide with the centre of the main component. The second component then falls inside the aperture corresponding to $R_{500}$ (at a distance from the main cluster of $\sim 5-7$ arcmin, where $R_{500}$ corresponds to $11.1 \mathrm{arcmin}$ ) and contributes to the substructure measure ${ }^{4}$.

\footnotetext{
${ }^{4}$ Note that for the determination of the global cluster properties (Pratt et al. 2009) and density and temperature profiles (Croston et al. 2008; Pratt et al. 2007, 2010) this second component was excised.
} 


\subsection{Image analysis and scaling}

The treatment of the X-ray data and production of the images is described in Böhringer et al. (2007) and images for all clusters are displayed in Pratt et al. (2009). We make use exclusively of the 0.5 to $2.0 \mathrm{keV}$ energy band, since this is approximately the energy range providing X-ray images with the highest signalto-noise. We combine the data from the three detectors into one composite image which has a minimal number of pixels with zero exposure. Before combining the images, the background is subtracted. The background is obtained from a model fit to a source excised blank sky field, where the model includes homogeneous vignetted and unvignetted components. Its normalisation is obtained for each detector separately from a comparison to the surface brightness in the outer cluster-free region. To properly combine the images of the three detectors, we scale the exposure time of the EMOS data sets to the sensitivity ratio between the EPN and EMOS detectors (typically a factor of 3.3). The sensitivity ratio is obtained directly from the data by determining the scaling of the cluster surface brightness profiles observed with the three detectors. Scaling the exposure map and keeping the summed count image preserves the information on the photon statistics that will become necessary in the course of the analysis. Thus the resulting combined exposure map is in units of effective EPN exposure time. Additional $\mathrm{X}$-ray sources in the field-of-view are identified and their extent evaluated using the wavelet-based $\mathrm{SAS}^{5}$ routine ewavdetect. The outcome is visually inspected to distinguish significant point sources from extended cluster substructure emission. Non-point sources which correspond to substructure features in the cluster are deleted from the source list. The sources are then excised from the photon data, with visual checking if the excision radii are sufficient or have to be increased manually. The holes are then filled with the Chandra $\mathrm{CIAO}^{6}$ routine dmfilth using randomisation based on the surface brightness distribution around the holes to fill the gap. The analysis methods described below are then applied to these cosmetically point source-cleaned cluster images. The total area replaced is in all cases a very small fraction of the total image, so that the effect of this cosmetic operation on the photon statistics can be neglected. Excision of the point sources is necessary, however, since their presence near the aperture boundary can severely affect the results.

The total number of photons detected per cluster in all three detectors combined ranges from 30000 to 170000 source photons inside $R_{500}$, except for two observations with fewer photons that we used as split observations (more details in Appendix A.1), and for A1689 (RXC J1311.4-0120), for which more than 300000 photons were observed. We therefore have very good photon statistics with which to characterise the cluster morphology. In the following analysis we sample the surface brightness inside a radius of $R_{500}$, which is defined as the radius inside which the mean total mass density in the cluster is 500 times the critical density of the Universe. The value of $R_{500}$ is not estimated directly from the $\mathrm{X}$-ray derived mass profile, but from the correlation of $M_{500}$ with $Y_{\mathrm{X}}$ (see also Pratt et al. 2009). The latter parameter, which is the product of the gas mass inside $R_{500}$ and the mean spectroscopic temperature derived from the photons in the radial range $[0.15-0.75] R_{500}$, has

\footnotetext{
5 SAS is the ESA provided analysis software system for the reduction of XMM-Newton observational data. Information can be found at: http: //xmm.vilspa.esa.es/sas/.

6 CIAO is the publically provided analysis software system for the reduction of Chandra observational data. Information can be found at: http: //cxc . harvard. edu/ciao/.
}

been found to be a low-scatter mass proxy in numerical simulations (Kravtsov et al. 2006). Its low scatter has been confirmed in the observational analysis of e.g. Arnaud et al. (2007). $M_{500}$ is found by iteration about the relation given by Arnaud et al. (2007), as described by Kravtsov et al. (2006). $R_{500}$ not only characterises the part of the cluster that is fairly virialised (in non-merging systems) and thus less affected by the matter infall region (e.g. Evrard et al. 1996), but it also marks the region inside which we have a highly significant detection of the X-ray surface brightness for all systems. Therefore this fiducial radius lends itself naturally as the outer limiting radius for our study.

In the following we use two methods for the substructure characterisation. One - the so-called power ratio method - is based on a multipole analysis of the azimuthal surface brightness distribution (Buote \& Tsai 1995, 1996; Buote 2002; Jeltema et al. 2005, 2008; Valdarnini 2006). The other method is based on studying the emission centroid shift as a function of the integration radius (Mohr et al. 1993, 1995; Poole et al. 2006; O'Hara et al. 2006; Kay et al. 2007; Ventimiglia et al. 2008; Yang et al. 2008; Maughan et al. 2008).

\subsection{Power ratios}

The power ratio method introduced by Buote \& Tsai (1995) is motivated by the idea of identifying the X-ray surface brightness as a representation of the projected mass distribution of the cluster. The power ratio is then a multipole decomposition of the potential of the two-dimensional, projected mass distribution. Following the recipe of Buote $\&$ Tsai, the moments, $P_{n}$ are determined as follows

$$
\begin{aligned}
& P_{0}=\left[a_{0} \ln \left(R_{\mathrm{ap}}\right)\right]^{2} \\
& P_{m}=\frac{1}{2 m^{2} R_{\mathrm{ap}}^{2 m}}\left(a_{m}^{2}+b_{m}^{2}\right)
\end{aligned}
$$

where $R_{\text {ap }}$ is the aperture radius. The moments $a_{m}$ and $b_{m}$ are calculated using:

$a_{m}(R)=\int_{R^{\prime} \leq R_{\mathrm{ap}}} S\left(x^{\prime}\right)\left(R^{\prime}\right)^{m} \cos \left(m \phi^{\prime}\right) \mathrm{d}^{2} x^{\prime}$

and

$b_{m}(R)=\int_{R^{\prime} \leq R_{\text {ap }}} S\left(x^{\prime}\right)\left(R^{\prime}\right)^{m} \sin \left(m \phi^{\prime}\right) \mathrm{d}^{2} x^{\prime}$,

where $S(x)$ is the X-ray surface brightness, $x$ is used as a label for the pixel, and the integral extends over all pixels inside the aperture radius. $a_{0}$ in Eq. (1) is thus the total radiation intensity inside the aperture radius.

Since all terms $P_{m}$ are proportional to the total intensity of the cluster X-ray emission, while only the relative contribution of the higher moments to the total emission is of interest, the multipole expansion power terms are normalised by $P_{0}$, resulting in the so-called power ratios, $P_{m} / P_{0}$. Similarly to all previous studies, we only make use of the lowest moments from $P_{1}$ to $P_{4}$ (the dipole, quadrupole, hexapole and octopole). The dipole, $P_{1}$, is used to find the centre of symmetry of the X-ray surface brightness distribution. We use a minimisation routine (the simplex method of Press et al. 1992) to find the minimum of $P_{1}$ as a function of input centre coordinates. The disappearance of the dipole indicates that the signal is well balanced in opposite directions from the centre at all position angles, and we take this as a sign that the cluster is correctly centred (with respect to the 
Table 1. Substructure parameters for 31 clusters from the REXCESS sample.

\begin{tabular}{|c|c|c|c|c|c|c|c|c|c|c|c|}
\hline Cluster & $\begin{array}{r}P 2 / P 0 \\
\times 10^{-5} \\
(2)\end{array}$ & $\begin{array}{c}\text { Bias } \\
\times 10^{-5} \\
\end{array}$ & $\begin{array}{r}\text { Error } \\
\times 10^{-5} \\
(4)\end{array}$ & $\begin{array}{r}P 3 / P 0 \\
\times 10^{-7} \\
\quad(5)\end{array}$ & $\begin{array}{r}\text { Bias } \\
\times 10^{-7} \\
(6)\end{array}$ & $\begin{array}{r}\text { Error } \\
\times 10^{-7} \\
(7)\end{array}$ & $\begin{array}{r}P 4 / P 0 \\
\times 10^{-7} \\
\quad(8)\end{array}$ & $\begin{array}{r}\text { Bias } \\
\times 10^{-7} \\
\quad(9)\end{array}$ & $\begin{array}{r}\text { Error } \\
\times 10^{-7} \\
(10)\end{array}$ & (11) & Error \\
\hline RXC J0003.8+0203 & 0.205 & 0.0066 & 0.050 & 0.337 & 0.1923 & 0.379 & 0.5507 & 0.083 & 0.334 & 0.0032 & 0.00102 \\
\hline RXC J0006.0-3443 & 0.546 & 0.0088 & 0.091 & 2.303 & 0.2807 & 1.322 & 0.2767 & 0.127 & 0.281 & 0.0130 & 0.00144 \\
\hline RXC J0020.7-2542 & 0.135 & 0.0049 & 0.036 & -0.139 & 0.1777 & 0.372 & 0.3431 & 0.069 & 0.323 & 0.0063 & 0.00078 \\
\hline RXC J0049.4-2931 & 0.148 & 0.0106 & 0.069 & 0.295 & 0.3261 & 0.851 & 1.8380 & 0.160 & 0.922 & 0.0023 & 0.00078 \\
\hline RXC J0145.0-5300 & 1.252 & 0.0072 & 0.132 & 1.155 & 0.2146 & 0.787 & 1.9030 & 0.093 & 0.700 & 0.0300 & 0.00141 \\
\hline RXC J0211.4-4017 & 0.523 & 0.0096 & 0.112 & -0.223 & 0.3101 & 0.358 & 2.3980 & 0.136 & 0.782 & 0.0046 & 0.00100 \\
\hline RXC J0225.1-2928 & 0.896 & 0.0238 & 0.205 & 7.652 & 0.7239 & 3.654 & 1.9670 & 0.354 & 1.300 & 0.0121 & 0.00136 \\
\hline RXC J0345.7-4112 & 0.366 & 0.0120 & 0.073 & 3.363 & 0.3813 & 1.735 & 2.1840 & 0.179 & 0.918 & 0.0052 & 0.00088 \\
\hline RXC J0547.6-3152 & 0.112 & 0.0028 & 0.021 & 1.822 & 0.0699 & 0.535 & 1.0220 & 0.035 & 0.235 & 0.0070 & 0.00057 \\
\hline RXC J0605.8-3518 & 0.155 & 0.0026 & 0.027 & 0.009 & 0.0745 & 0.114 & 0.0690 & 0.034 & 0.073 & 0.0059 & 0.00039 \\
\hline RXC J0616.8-4748 & 0.461 & 0.0079 & 0.110 & 7.580 & 0.2327 & 2.183 & 3.6510 & 0.106 & 0.900 & 0.0131 & 0.00151 \\
\hline RXC J0645.4-5413 & 0.416 & 0.0036 & 0.059 & -0.110 & 0.1084 & 0.159 & 0.2678 & 0.051 & 0.181 & 0.0039 & 0.00042 \\
\hline RXC J0821.8+0112 & 0.207 & 0.0253 & 0.109 & 9.254 & 0.8668 & 4.260 & 2.4590 & 0.372 & 1.617 & 0.0045 & 0.00144 \\
\hline RXC J0958.3-1103 & 0.211 & 0.0063 & 0.048 & 0.038 & 0.1830 & 0.357 & 0.2421 & 0.086 & 0.203 & 0.0034 & 0.00072 \\
\hline RXC J1044.5-0704 & 0.321 & 0.0021 & 0.031 & -0.035 & 0.0613 & 0.055 & -0.0092 & 0.027 & 0.051 & 0.0072 & 0.00040 \\
\hline RXC J1141.4-1216 & 0.098 & 0.0033 & 0.023 & 1.129 & 0.0917 & 0.457 & 0.1163 & 0.040 & 0.136 & 0.0054 & 0.00059 \\
\hline RXC J1236.7-3354 & 0.038 & 0.0086 & 0.030 & 0.227 & 0.2701 & 0.620 & 0.2089 & 0.117 & 0.418 & 0.0052 & 0.00069 \\
\hline RXC J1302.8-0230 & 1.233 & 0.0071 & 0.129 & 3.304 & 0.2152 & 1.100 & 1.3020 & 0.094 & 0.553 & 0.0153 & 0.00086 \\
\hline RXC J1311.4-0120 & 0.035 & 0.0009 & 0.007 & 0.041 & 0.0206 & 0.051 & 0.0121 & 0.009 & 0.020 & 0.0040 & 0.00029 \\
\hline RXC J1516.3+0005 & 0.099 & 0.0035 & 0.023 & 0.415 & 0.1030 & 0.328 & 0.3115 & 0.043 & 0.187 & 0.0037 & 0.00044 \\
\hline RXC J1516.5-0056 & 0.636 & 0.0075 & 0.091 & 10.250 & 0.2445 & 2.058 & 1.0280 & 0.106 & 0.479 & 0.0176 & 0.00140 \\
\hline RXC J2014.8-2430 & 0.073 & 0.0020 & 0.015 & 0.614 & 0.0597 & 0.300 & 0.1227 & 0.026 & 0.093 & 0.0058 & 0.00030 \\
\hline RXC J2023.0-2056 & 0.056 & 0.0155 & 0.051 & -0.338 & 0.5603 & 0.610 & 0.8529 & 0.252 & 0.809 & 0.0167 & 0.00147 \\
\hline RXC J2048.1-1750 & 0.840 & 0.0051 & 0.080 & 3.638 & 0.1479 & 0.866 & 2.0140 & 0.066 & 0.456 & 0.0419 & 0.00432 \\
\hline RXC J2129.8-5048 & 0.037 & 0.0079 & 0.090 & 2.966 & 0.2349 & 1.220 & -0.0584 & 0.107 & 0.269 & 0.0419 & 0.01997 \\
\hline RXC J2149.1-3041 & 0.011 & 0.0046 & 0.012 & 1.951 & 0.1294 & 0.685 & 0.0450 & 0.059 & 0.082 & 0.0034 & 0.00053 \\
\hline RXC J2157.4-0747 & 1.501 & 0.0266 & 0.275 & -0.847 & 0.9897 & 0.954 & 3.4010 & 0.444 & 2.032 & 0.1080 & 0.90430 \\
\hline RXC J2217.7-3543 & 0.061 & 0.0037 & 0.022 & 0.705 & 0.1116 & 0.364 & 0.2425 & 0.046 & 0.203 & 0.0018 & 0.00373 \\
\hline RXC J2218.6-3853 & 0.762 & 0.0022 & 0.052 & 0.514 & 0.0728 & 0.287 & 0.1893 & 0.028 & 0.099 & 0.0155 & 0.00049 \\
\hline RXC J2234.5-3744 & 0.144 & 0.0040 & 0.027 & -0.085 & 0.1166 & 0.181 & 0.3276 & 0.055 & 0.148 & 0.0075 & 0.00056 \\
\hline RXC J2319.6-7313 & 0.694 & 0.0089 & 0.138 & -0.198 & 0.3012 & 0.799 & 0.5018 & 0.126 & 0.346 & 0.0217 & 0.00085 \\
\hline
\end{tabular}

Notes. For this analysis the central cluster emission inside a radius of $0.1 \times R_{500}$ was excised to avoid any influence of cluster cool cores. The power ratio parameters have been determined for an aperture with a radius of $R_{500}$. The corresponding results without center excision are given in Table A.1 in the Appendix. For each of the power ratio parameters we provide the value of the noise contribution to the power ratio result (bias) which has been subtracted from the measured result to provide the value listed in Cols. 2, 5, and 8. The uncertainties determined from the Poissonisation simulations are listed in Cols. 4, 7, and 10 (error). The center shift statistic parameter $w$ and its uncertainty are listed in Cols. 11 and 12 .

radial weighting scheme used to calculate $P_{1}$ ). We checked visually that this defines the centre of symmetry, and most often also the central maximum of the cluster's X-ray emission, and found the method to be reliable. We then used this centre, defined for a vanishing dipole signal, to determine the power ratios from $P_{2} / P_{0}$ to $P_{4} / P_{0} . P_{2} / P_{0}$ describes the quadrupole of the surface brightness distribution and is not necessarily a measure of substructure; a quadrupole will also be detected for a very regular elliptical cluster, which could be well relaxed. In practice, low to moderate values of $P_{2} / P_{0}$ are found for regular elliptical clusters, while larger values of $P_{2} / P_{0}$ are a sign of cluster mergers. The lowest power ratio moment providing a clear substructure measure is thus $P_{3} / P_{0} . P_{4} / P_{0}$ describes substructure on slightly finer scales and is found to be correlated to $P_{2} / P_{0}$ in this and previous studies.

The results from the power ratio analysis determined within $R_{500}$ are given in Table 1 for core-excised cluster images and in Table A. 1 for the full aperture. In Table 2 we also give the cluster centres in sky coordinates, as determined from the dipole minimisation for the innermost aperture with no center excision. The tables also give two measures of the uncertainty on the power ratios, as we discuss below and in more detail in the Appendix.
To assess whether we have detected a significant deviation from azimuthal symmetry, we must consider the effect of photon noise. This noise first introduces a bias since even a completely symmetric cluster would be detected with some residual structure due to the photon noise affecting real observations. We estimate this bias from Monte Carlo simulations of azimuthally randomised cluster images, and subtract this signal from the power ratios. The statistical uncertainties of significant substructure signal, again due to photon noise, are then estimated from a second set of Monte Carlo simulations in which Poisson noise is added to the observational data and uncertainties are determined from standard deviations. These uncertainties are larger than the bias for clusters with significant substructure signal. These methods of error estimation are further explained in the Appendix, where we also quantify how realistic these estimated uncertainties are.

To explore the effects of cool cores in the clusters, we have undertaken the power ratio analysis both with and without excising the central region $\left(r \leq 0.1 R_{500}\right)$. From the above power ratio definition it is obvious that for large values of $R_{\text {ap }}$ the exclusion of the central region has little influence on the magnitude of the derived power ratios. The question of whether the central regions 
Table 2. Coordinates (J2000) of the cluster centers for 31 objects of the REXCESS cluster sample.

\begin{tabular}{|c|c|c|c|c|c|}
\hline \multirow[t]{2}{*}{ Cluster } & \multicolumn{2}{|c|}{ Local maximum } & \multicolumn{2}{|c|}{$P_{1}$ minimum } & \multirow[t]{2}{*}{$F$} \\
\hline & RA & Dec & RA & Dec & \\
\hline$\overline{\mathrm{R} 0003+0203}$ & 000349.7 & 020357.5 & 000349.6 & 020351.9 & 1 \\
\hline R0006-3443 & 000559.9 & -344323.0 & 000600.4 & -344319.3 & 1 \\
\hline R0020-2542 & 002042.2 & -254224.5 & 002041.9 & -254236.0 & 1 \\
\hline R0049-2931 & 004923.0 & -293113.9 & 004923.0 & -293111.8 & 1 \\
\hline R0145-5300 & 014459.7 & -530103.1 & 014458.1 & -530112.9 & 1 \\
\hline R0211-4017 & 021124.8 & -401728.3 & 021125.1 & -401728.4 & 1 \\
\hline R0225-2928 & 022509.3 & -292836.3 & 022508.5 & -292836.4 & 1 \\
\hline R0345-4112 & 034546.2 & -411213.8 & 034546.4 & -411215.7 & 1 \\
\hline R0547-3152 & 054738.4 & -315212.2 & 054738.5 & -315210.9 & 1 \\
\hline R0605-3518 & 060554.2 & -351808.7 & 060554.0 & -351808.3 & \\
\hline R0616-4748 & 061651.7 & -474740.4 & 061651.5 & -474742.2 & \\
\hline R0645-5413 & 064529.3 & -541340.4 & 064529.4 & -541338.9 & \\
\hline $\mathrm{R} 0821+0112$ & 082150.9 & 011152.4 & 082150.7 & 011156.1 & \\
\hline R0958-1103 & 095822.3 & -110353.6 & 095822.1 & -110350.3 & \\
\hline R1044-0704 & 104433.0 & -070408.6 & 104432.9 & -070407.7 & 1 \\
\hline R1141-1216 & 114124.4 & -121637.4 & 114124.4 & -121638.8 & 1 \\
\hline R1236-3354 & 123641.3 & -335537.2 & 123641.0 & -335526.3 & 1 \\
\hline R1302-0230 & 130253.3 & -023100.4 & 130252.7 & -023056.9 & 1 \\
\hline R1311-0120 & 131129.5 & -012027.7 & 131129.4 & -012030.1 & 1 \\
\hline R1516+0005 & 151618.1 & 000527.8 & 151618.0 & 000523.1 & 1 \\
\hline R1516-0056 & 151644.2 & -005811.9 & 151644.5 & -005821.8 & 1 \\
\hline R2014-2430 & 20 & -24 & 2014 & -243021.6 & 1 \\
\hline 056 & 202258.8 & 56.1 & 202259.0 & -205657.2 & 1 \\
\hline R2048-1750 & 2048 & -175 & 204810.4 & -175035.1 & \\
\hline R2129-5048 & 212936.5 & -504852.2 & 212940.9 & -504854.8 & \\
\hline R2149-3041 & 214907.6 & -304204.7 & 214907.6 & -304204.6 & \\
\hline R2157-0747 & _ & & 215729.5 & -074754.9 & \\
\hline R2217-3543 & 221745.5 & -354330.1 & 221745.6 & -354331.0 & \\
\hline R2218-3853 & 221840.3 & -385405.7 & 221840.1 & -385400.6 & \\
\hline R2234-3744 & 223428.0 & -374352.1 & 223427.1 & -374402.3 & \\
\hline R2319-7313 & 231940.2 & -73 1338.4 & 231939.9 & -73 1336.1 & \\
\hline
\end{tabular}

Notes. The first set of coordinates designate the local X-ray maximum found in X-ray images $(0.5-2 \mathrm{keV}$ band) smoothed with a Gaussian with $\sigma \sim 8$ arcsec. The second set of coordinates is derived from minimising the dipole $\left(P_{1}\right)$ signal in the determination of the power ratios in the smallest aperture with a radius of $0.1 \times R_{500}$. The flag, F, indicates which cluster centre has been used for the determination of the centre shift parameter $w$. For flag 1 the local maximum of the smoothed images was used (default), for clusters with flag 0 the P1 minimisation result was used.

are retained or excised is more important for the second method of structure assessment described in the next section.

\subsection{Centre shifts}

The centre shift method is based on a measurement of the variation in separation between the X-ray peak and the centroid calculated within an increasing aperture size. For our analysis we use two centre definitions: the centres found in the $P_{1}$ minimisation procedure described above, and centres found by the determination of a local maximum in smoothed images ${ }^{7}$. The latter is determined from the X-ray surface brightness peak on an image smoothed with a Gaussian with $\sigma$ of $\sim 8$ arcsec (in images where point sources have been removed). We determine the centre shift

\footnotetext{
7 We have also used manually determined local maxima and the maximum obtained from minimisation of $P_{1}$ in the innermost region $(r<$ $\left.0.1 R_{500}\right)$. The results we obtained from the different approaches are quantitatively similar and demonstrate the robustness of the centre shift parameters obtained.
}

between the local maximum and the centroids obtained by $P_{1}$ minimisation within 10 apertures $\left(r \leq n \times 0.1 R_{500}\right.$, with $n=1$, $2 \ldots 10)$. For the runs where the central region $\left(r \leq 0.1 R_{500}\right)$ is excised, we have centroids within 9 apertures. The fiducial $w$ parameter is then the standard deviation of the different centre shifts (in units of $R_{500}$ ), defined as (see also Poole et al. 2006):

$$
w=\left[\frac{1}{N-1} \sum\left(\Delta_{i}-\langle\Delta\rangle\right)^{2}\right]^{1 / 2} \times \frac{1}{R_{500}}
$$

where $\Delta_{i}$ is the distance between the X-ray peak and the centroid of the $i$ th aperture.

The uncertainties in the centre shifts and in the $w$ parameter are determined with the same simulations as the uncertainties of the power ratios, i.e., by using Poissonised resampled cluster $\mathrm{X}$-ray images. The standard deviation of the $w$ parameter in the simulation results is used as an estimate of the measurement uncertainties. We do not subtract a noise bias for the $w$-parameter as in the case of the power ratios. The results of the centre shift analysis are given for core-excised cluster images in Table 1 and for the full aperture in Table A.1. Table 2 gives the cluster centres from the determination of the local maxima, in sky coordinates, as used in the centre shift analysis.

\section{Simulations}

The representative nature of REXCESS, and the homogeneous nature of the associated X-ray observations, makes it an ideal sample for comparison with numerical simulations. What interests us most here is the question of whether the simulated clusters resemble the clusters observed in our Universe, when the comparison is based on a representative sample of real clusters.

The set of simulated clusters that we use for this comparison comprises 117 clusters identified from the hydrodynamical simulation of a large cosmological volume in a $\Lambda \mathrm{CDM}$ model, presented by Borgani et al. (2004). These clusters have virial masses in the range $0.8 \times 10^{14}$ to $1.3 \times 10^{15} h^{-1} M_{\odot}$. As such, the sample covers a range of ICM emission weighted temperatures, $T_{\text {ew }}$, between 1.18 and $7.1 \mathrm{keV}$. Since there is only one cluster with a temperature larger than $5 \mathrm{keV}$, we supplemented the sample with four massive objects taken from the set of simulated clusters presented by Dolag et al. (2008). These additional systems have emission-weighted temperatures in the range $T_{\mathrm{ew}}=8.1-12.6 \mathrm{keV}$ and virial masses in the range 1.0 to $2.2 \times 10^{15} h^{-1} M_{\odot}$. The mass and ICM temperature distribution of the simulated clusters is thus different from that of the REXCESS sample and we discuss this in terms of a fair comparison below. All simulations have been carried out with the TreePM-SPH GADGET-2 code (Springel 2005). They included the treatment of radiative cooling, a uniform time-dependent UV background, and a sub-resolution model for star formation and energy feedback from galactic winds (see Springel \& Hernquist 2003 for details). All clusters are identified from the simulations at $z=0$.

$\mathrm{X}$-ray images for these clusters were produced and analysed by Ameglio et al. $(2007,2009)$. They have also been used for a similar sub-structure analysis in connection with the masstemperature relation of clusters by Ventimiglia et al. (2008). X-ray emission within each pixel has been computed by accounting for the contribution of all the gas particles falling in projection within that pixel, as described by Ameglio et al. (2007). The images used here have been obtained for the energy range 0.5 to $2 \mathrm{keV}$, which is the same energy band used for the analysis of XMM-Newton observations. The synthetic X-ray images do not include any X-ray background and photon noise, 

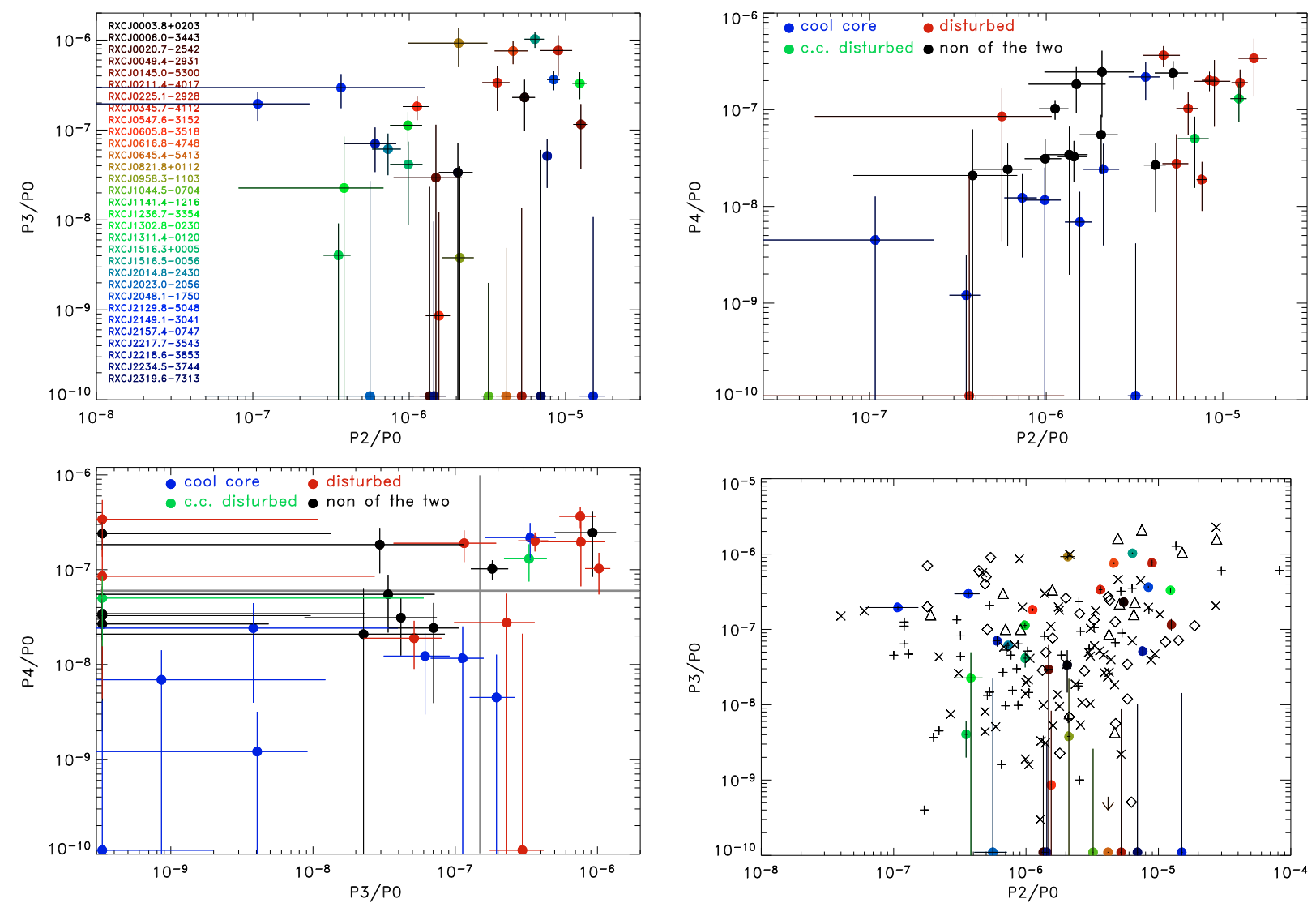

Fig. 1. Results of the power ratio analysis for the 31 clusters of the REXCESS sample, determined for photons the radial range [0.1-1] $R_{500}$. The upper left, upper right and lower left panels show the distribution of $P 2 / P 0, P 3 / P 0$, and $P 4 / P 0$ in pairs. The uncertainties shown are determined by Monte Carlo simulations with Poisson noise added to the image pixels. While in the upper left panel we identify the clusters by their name in colors, we use some cluster properties for the identification of the data points for the upper right and lower left panel (as used in Pratt et al. 2009, the threshold for a cluster to be considered as morphologically disturbed is a $\langle w\rangle$ parameter $\geq 0.01$ ). In the lower left panel we define a tentative classification for a cluster to be morphologically disturbed in terms of the $P 3 / P 0$ and $P 4 / P 0$ parameters, shown by the grey lines (numerical values given in the text). The lower right panel shows a comparison of our $\mathrm{P} 2 / \mathrm{P} 0$ and $\mathrm{P} 3 / \mathrm{P} 0$ parameter results and those from the literature. The diamonds and triangles are data from Jeltema et al. (2005) for clusters with $z<0.45$ and $z>0.45$, respectively. The $\times$ and + symbols correspond to the data from Buote \& Tsai (1996) for power ratios determined with apertures $R_{\text {ap }} \leq 0.7 h_{80}^{-1} \mathrm{Mpc}$ and $R_{\text {ap }}>0.7 h_{80}^{-1} \mathrm{Mpc}$, respectively. In this plot the uncertainties on the REXCESS data are calculated from the azimuthal randomization test (see Fig. A.1, and accompanying text).

thus the outcome of the analysis of the simulated images is interpreted as results without statistical uncertainties. For all aspects of the analysis carried out in this paper, this makes the interpretation easier and has no effect on the conclusions from the comparison with real data. The centre of each map of X-ray emissivity coincides in projection with the minimum of the cluster gravitational potential. Each image has a size of 256 by 256 pixels and covers a region of $4 \times R_{500}$ around each cluster. The simulated cluster images have a resolution of $\sim 0.031 R_{500}$, comparing well to the resolution of observations, which are characterised by a typical half energy width of $0.030-0.033 R_{500}$. As we discuss in the Appendix, the resolution at which the X-ray emission of real clusters is observed has no significant effect on the results of the substructure analysis at the range of resolution powers relevant here. Any difference in the resolution between simulated and REXCESS cluster maps is therefore not important for the purpose of our analysis.

\section{Results of the morphological analysis}

In this section we first present the observational results, then discuss some implications of the analysis of the simulations, and finally, we compare the observations to the simulations.

\subsection{Power ratios}

The first three panels in Fig. 1 show the results of the power ratio analysis in pairs of two of the power ratio parameters. As detailed above, values shown are those for an aperture of $R_{500}$ (with the central $0.1 R_{500}$ excised) so as to provide a global characterization of the clusters. While for the upper left and lower right panel the clusters are colour coded to identify them by their name, we characterise the clusters in the other two panels by their properties (cool cores, having central densities $h_{70}^{-2} n_{e 0}>4 \times 10^{-2} \mathrm{~cm}^{-3}$, and morphologically disturbed clusters, having a $w$ parameter $>0.01)^{8}$. The values of $P_{2} / P_{0}$ (i.e., the quadrupole moments) are larger than the higher order ratios. Even though the data have very good photon statistics compared to the average X-ray observation, the results have substantial photon noise uncertainties, implying that the power ratio method becomes insufficiently sensitive for images of much lower quality. The $P_{2} / P_{0}$ (quadrupole) versus $P_{4} / P_{0}$ (octople) plot shows, as in previous studies, the strongest correlation. However, the plot of $P_{3} / P_{0}$ vs. $P_{4} / P_{0}$ also shows a correlation, such that both

8 These classifications were used Pratt et al. (2009) to isolate the most extreme thirty per cent of the sample according to those criteria, and are further discussed in Sect. 4.2. 
parameters will most likely flag the same cluster as either regular or disturbed. With respect to the cool core and morphological disturbance criteria mentioned above, we note that in both the $P_{2} / P_{0}-P_{4} / P_{0}$ pair and the $P_{3} / P_{0}-P_{4} / P_{0}$ pair most clusters designated as disturbed accumulate in the upper right quadrant and most cool core clusters accumulate in the lower left quadrant.

We now try to set a tentative threshold criterion for the dynamical state of a system in terms of the power ratio parameters $P_{3} / P_{0}$ and $P_{4} / P_{0}$. We find that a value of $P_{3} / P_{0}=1.5 \times 10^{-7}$ separates out 11 non-symmetric clusters (about one third of the sample), and a value of $P_{4} / P_{0}=6 \times 10^{-8}$ separates out 13 clusters. These threshold criteria are shown as horizontal and vertical grey lines in the lower left panel of Fig. 1.

The three clusters with no significant $P_{3} / P_{0}$ but high $P_{4} / P_{0}$ are, from top to bottom in the plot: RXC J2157.4-0747, RXC J0211.4-4017, and RXC J2023.0-2056. RXC J2157.40747 is the double component cluster which features a large centre shift and large quadrupole and octopole moments, but just lacks a hexapole moment. RXC J0221.4-4017 appears rather regular but obtains a significant octopole moment due to some distortion in the outer surface brightness contour. The third cluster in this group, RXC J2023.0-2056 is azimuthally symmetric on large scale but has an Eastern extension of the central region.

Similarly we find three clusters separated out by the $P_{3} / P_{0}$ parameter as disturbed but having a low $P_{4} / P_{0}$ value: RXC J0006.0-3443, RXC J2149.1-3041, and RXC J2129.85048. The first cluster has no significant octopole moment, the second cluster is fairly regular but displays a significant hexapole moment due to some distortions in the outer surface brightness contour, and RXC J2129.8-5048 has a low surface brightness elongation to the NE but no significant octopole moment. One may thus consider a combination of $P_{3} / P_{0}$ and $P_{4} / P_{0}$ measures, with the threshold criteria defined above, as a good general characterisation of significant substructure in a given system. However, distortions near the aperture radius can have a strong influence on the derived power ratios.

\subsection{Centre shifts}

The $w$-parameters found for the REXCESS sample are given in Table 1, and the centre coordinates used for this analysis are compared to those obtained from the dipole minimisation in Table 2. The two centres are generally very similar. Exceptions are the three clusters with very diffuse, low surface brightness regions in the centre, RXC J2048.1-1750, RXC J2129.8-5048, and RXC J2157.4-0747. Here the local maximum is difficult to define automatically, even with smoothing, and the minimisation of the dipole moment marks a much more reliable central location as determined by visual inspection of the images. While in the first two cases the offset is moderate, for RXC J2157.4-0747 the centre determination is not stable, and we do not give results for the local maximum for this cluster. Therefore we recommend minimisation of the dipole moment to determine the centre for these and similar systems, as indicated by the flag in Table 2 . In addition, for RXC J2234.5-3744, which shows some substructure in the central region, the dipole moment centring provides a better indication of the local maximum.

\subsection{Comparison with previous work}

In the lower right panel of Fig. 1 we compare the range of our results for the $P_{2} / P_{0}$ versus $P_{3} / P_{0}$ correlation to the previous observational results from Buote \& Tsai (1996) and
Jeltema et al. (2005). We note that the parameter range covered is very similar for all the studies. Some of the data points from the work of Jeltema et al. extend to somewhat higher values in both parameters, as indicated by the points in the upper right corner. However, for these points no photon noise bias was subtracted, as it was for our data, and some of the clusters in the Jeltema et al. sample have large photon noise and would possibly have significantly reduced values if the photon noise correction were to be applied. Therefore we conclude that the parameter range covered by observational studies up to date is very similar, even though the selection of the cluster sample is not strictly equivalent.

Maughan et al. (2008) have studied a large sample of 115 galaxy clusters in the redshift range 0.1 to 1.3 observed with Chandra, and analysed the cluster morphologies with the same centre shift parameter technique as the one used in the present work. They find a very similar distribution of $w$ parameter values, lying in the range $0.0007-0.0695$.

\subsection{Comparison of power ratios and centre shifts}

\subsubsection{Sorting clusters}

The clusters marked in red in the lower left hand panel of Fig. 1 were identified as the thirty per cent of the sample that were most disturbed according to the $w$ parameter (see Pratt et al. 2009 and Sect. 4.2). Of these, only one cluster, RXC J2218.63853 , has no large hexapole or octopole moment; however, this system is elongated with a significant quadrupole moment and has a large isophotal centre shift in the central region. In addition, two cool core clusters, RXC J1302.8-0230 (green) and RXC J0345.7-4112 (blue) are among the clusters classified as disturbed through the $P_{3} / P_{0}$ vs. $P_{4} / P_{0}$ classification. While RXC J0345.7-4112 shows a very regular central region, it has a very low surface brightness extension in its Eastern outer regions; RXC J1302.8-0230 has a cool core but with a clear offset from the overall cluster symmetry.

In Fig. 2 we plot $w$ versus $P_{3} / P_{0}$ for the 31 clusters of the REXCESS sample ${ }^{9}$. We have selected the $P_{3} / P_{0}$ parameter for this comparison since it is the lowest multipole moment that gives an unambiguous signature of dynamical distortion (since an elliptical cluster can be relaxed). While there is a clear correlation between the two parameters, there is also a large scatter, illustrating that the two methods are weighing structural features in different ways. The horizontal and vertical lines in the upper panel $\left(P_{3} / P_{0}=4 \times 10^{-8} ; w=0.006\right)$ divide the sample roughly in half with respect to the two parameters, with fifteen objects in the lower (left) half and sixteen in the upper (right) half of the figure. Alternatively, using the larger gap in the $w$-parameter distribution ( $w=0.007-0.01$, marked by a grey stripe in the figure), we find that 21 clusters are found in the lower left and upper right quadrants, while only 10 clusters fall near the boundaries into the other two quadrants, which implies that with these dividing lines $68 \%$ of the clusters would be classified in the same way and $32 \%$ differently using these parameter cuts.

Inspecting the clusters which have discrepant substructure classifications in terms of $P_{3} / P_{0}$ and $w$ parameter (Fig. 2), we easily find the reason for the discrepancy. The three clusters in the upper left of Fig. 2, having no significant value of $P_{3} / P_{0}$, are: RXC J2157.4-0747 (at the top) which is the double component

\footnotetext{
9 For the results plotted in the figure, the central region was excised, but including the central region gives very similar results (cf. corresponding Table A.1 in the Appendix).
} 

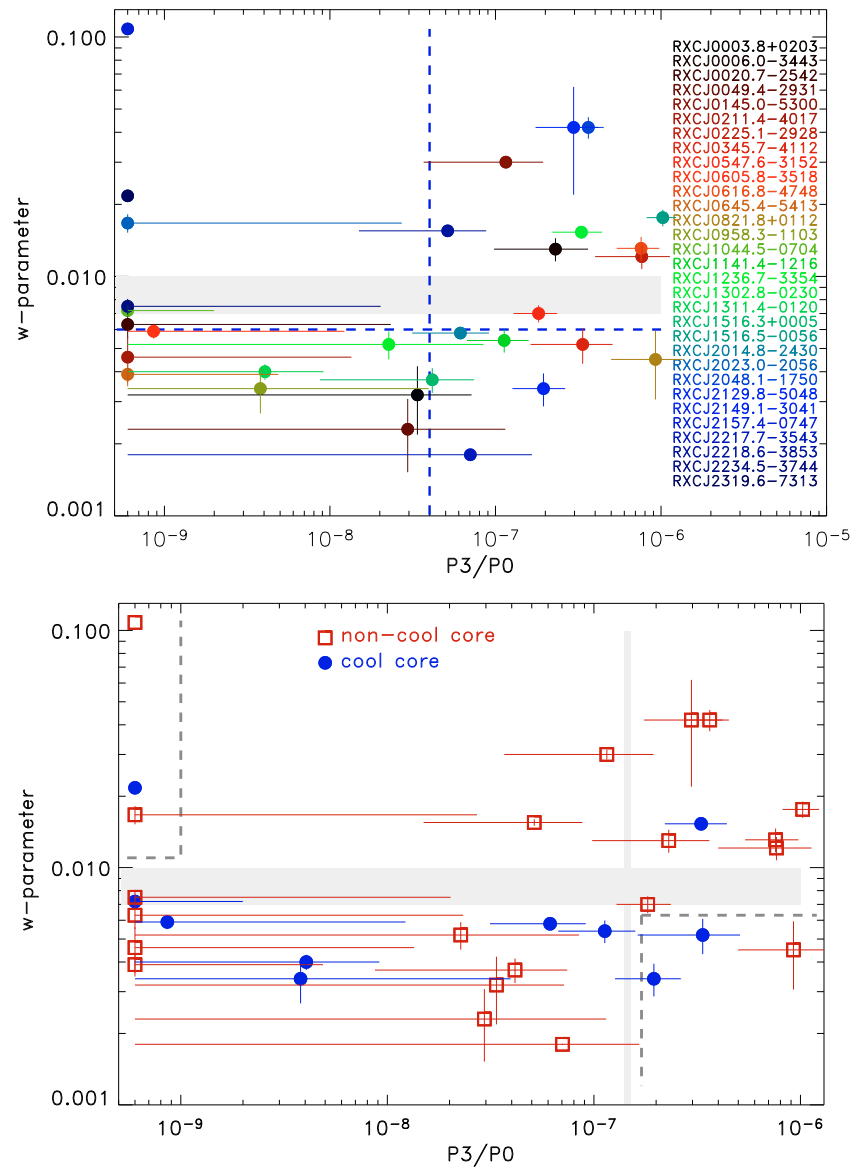

Fig. 2. Correlation of the $w$ parameter with $P 3 / P 0$ for the 31 clusters of the REXCESS sample, with both parameters derived from core excised images. Upper panel: clusters identified by name. The dashed blue lines divide the sample roughly in two halves with respect to both parameters. Lower panel: cool core clusters identified. The thin grey line marks the tentative $P 3 / P 0$ threshold criterion for morphological disturbance as defined in Fig. 1. The dashed grey lines enclose the clusters with discrepant power ratio vs. $w$ classification, as further discussed in the text. In both panels the grey stripe marks the gap in the $w$-parameter distribution used for further discussion of the distribution statistics in the text.

cluster which features a large centre shift and large quadrupole and octopole moments, but lacks a hexapole moment. Similarly, RXC J2319.6-7313 is elliptical in large scale morphology, with a bright, elongated central region, resulting in a strong centre shift and dipole moment but vanishing hexapole moment. RXC J2023.0-2056, is azimuthally symmetric on large scale but has an Eastern extension of the central region. Among the outliers above the $P_{3} / P_{0}$-threshold in the lower right corner of Fig. 2, RXC J0821.8+0112 is regular in the central region except for a substructure clump near $R_{500}$, RXC J0345.7-4112 shows a very regular central region, but has an very low surface brightness extension in the Eastern outer region, and RXC J2149.13041 is quite regular but distorted near $R_{500}$ as discussed above.

In the lower panel of Fig. 2 we designate the clusters by their cool core properties. While only two cool core clusters, RXC J1302.8-0230 and RXC J2319.6-7313, are classified by the $w$ parameter as disturbed, several cool core clusters are found to have high $P_{3} / P_{0}$ values. The two cool core clusters with the largest values of $P_{3} / P_{0}$ are RXC J0345.7-4112 and RXC J1302.8-0230 which have already been discussed above. As already noted, the difference in the classification obviously

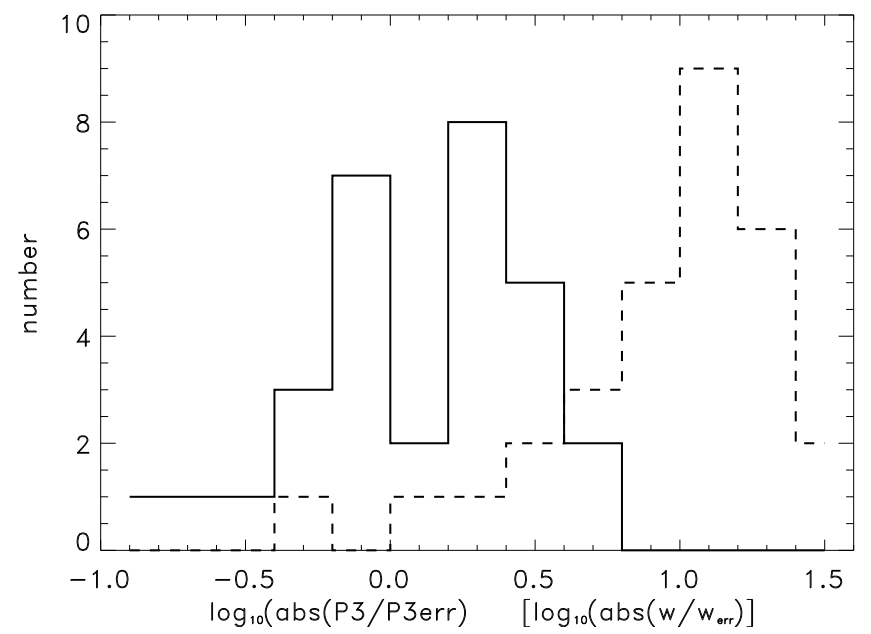

Fig. 3. Signal-to-noise $(P 3 / \Delta(P 3))$ and $(w / \Delta w)$ distribution of the power ratios (solid line) and centre shifts (dashed line) for the 31 REXCESS clusters (plotted in logarithmic units).

stems from the stronger weighting that the power ratios give to the outer regions. The $P_{3} / P_{0}$ parameter is very sensitive to substructure in the outskirts, while the centre shift parameter is very sensitive to isophotal structure in the inner region. This result is further explored in Sect. A.3 in the Appendix, where we study power ratios with varying aperture radius.

\subsubsection{Sensitivity}

The uncertainties derived from the Monte Carlo simulations for the $w$-parameter are in most cases smaller than the corresponding uncertainties for $P_{3} / P_{0}$. In particular, a useful signal/noise value is still obtained for small values of $w$, in contrast to the results for $P_{3} / P_{0}$. The log-mean relative errors for $P_{2}$ are 22 per cent (excluding 1 cluster with a result consistent with zero) and for $P_{3}$ they are 70 per cent (excluding the 8 clusters with signal less than zero). For the $w$ parameter the log-mean error is 15 per cent for all clusters. To evaluate the sensitivity of the method the uncertainties should be considered in the context of the dynamic range of the parameter values. Since for both $P_{3} / P_{0}$ and $w$ the values span a range of about two orders of magnitude $\left(10^{-8}-10^{-6}\right.$ for $P_{3} / P_{0}$ and $0.001-0.1$ for $\left.w\right)$, the relative errors can directly be compared. Figure 3 illustrates the significance of both methods: the typical uncertainty for $P_{3} / P_{0}$ is comparable to the parameter value, while for $w$ it is more of the order of 10 per cent. Such a comparison clearly shows that $w$ appears to be more sensitive than $P_{3} / P_{0}$.

The $w$ parameter also gives a better overall measure of the deviation from symmetry and is not discriminating against the central regions as do the power ratios (as illustrated by the examples discussed above). Hence we decided that the $w$ parameter better suits the goal of substructure characterisation in the REXCESS project, and we have made use of this substructure measure in our other REXCESS papers. Our practical approach to separating the disturbed clusters from the regular objects takes advantage of the observed gap in the $w$ parameter distribution around $w=0.01$. We designate clusters above this limit as disturbed, leaving 12 clusters with the classification of being dynamically distorted - about one third of the sample.

To quantify the correlation of the two substructure measures we analyse the correlation statistics of the data by means of the Kendall and Spearman tests. To estimate the test statistics 
Table 3. Correlation tests of various substructure measures using the 31 galaxy clusters in the REXCESS sample.

\begin{tabular}{lccrrr}
\hline \hline Correlation & $\tau$ & \multicolumn{1}{c}{$P$} & $\rho$ & \multicolumn{1}{c}{$P$} & Figure \\
$(1)$ & $(2)$ & $(3)$ & $(4)$ & $(5)$ & $(6)$ \\
\hline$P_{3} / P_{0}-w\left(R_{500}\right)$ & 0.80 & 0.43 & 0.21 & 0.25 & 2 \\
$P_{3} / P_{0}-w\left(R_{500}\right)^{a}$ & 1.22 & 0.22 & 0.29 & 0.12 & 2 \\
$P_{3} / P_{0}-w\left(0.9 R_{500}\right)$ & 1.63 & 0.10 & 0.29 & 0.10 & - \\
$P_{3} / P_{0}-w\left(0.8 R_{500}\right)$ & 2.20 & 0.03 & 0.37 & 0.044 & - \\
$P_{3} / P_{0}-w\left(0.7 R_{500}\right)$ & 2.13 & 0.03 & 0.38 & 0.035 & $\mathrm{~A} .5$ \\
$P_{3} / P_{0}-w\left(0.6 R_{500}\right)$ & 1.38 & 0.17 & 0.25 & 0.17 & - \\
$P_{3} / P_{0}-w\left(0.5 R_{500}\right)$ & 0.36 & 0.72 & 0.01 & 0.99 & - \\
$M_{500}-w$ & 0.24 & 0.81 & -0.04 & 0.81 & 11 \\
$M_{500}-P_{3} / P_{0}$ & 0.39 & 0.69 & -0.06 & 0.73 & 11 \\
$L_{1}-w$ & 0.49 & 0.63 & -0.09 & 0.63 & 12 \\
$L_{1}-P 3 / P 0$ & 1.48 & 0.14 & -0.22 & 0.22 & 12 \\
$M_{500}-L_{\text {rat }}$ & 0.03 & 0.97 & -0.04 & 0.84 & 14 \\
$L_{1}-L_{\text {rat }}$ & 1.41 & 0.16 & 0.245 & 0.18 & 14 \\
$L_{2}-L_{\text {rat }}$ & 0.43 & 0.67 & 0.055 & 0.76 & 14 \\
$n_{e 0}-P 3 / P 0$ & 1.54 & 0.12 & -0.30 & 0.10 & 13 \\
$t_{\text {cool }}-P 3 / P 0$ & 1.86 & 0.06 & 0.34 & 0.06 & 13 \\
$n_{e 0}-w$ & 2.57 & 0.01 & -0.52 & 0.004 & - \\
$t_{\text {cool }}-w$ & 2.62 & 0.009 & 0.50 & 0.006 & - \\
\hline
\end{tabular}

Notes. Column (1) lists the correlation tested, (2) gives the parameter of Kendall's $\tau$ test and (3) the corresponding probability of a nullcorrelation, (4) gives the result for Spearman's rank test $\rho$ and (5) the corresponding probability and (6) gives the figure number that shows the correlation. For the correlation analysis the ASURV software package (Isobe et al. 1986) was used. ${ }^{(a)}$ Same correlation as in the first row but excluding the outlier object RXC J2157.4-0747. ${ }^{(b)} L_{1}$ is the X-ray luminosity in the $R_{500}$ aperture in the [0.1-2.4] $\mathrm{keV}$ band and $L_{2}$ refers to the core excised luminosity (values given in Pratt et al. 2009).

we use the analysis package ASURV (Astronomical Survival Statistics, Isobe et al. 1986), which tests for correlations in the presence of censored data. Table 3 lists the results of the correlation of the $w$ vs. $P_{3} / P_{0}$ parameter, which gives probabilities of 0.43 and 0.25 for no correlation according to Kandell's $\tau$ and Spearman's $\rho$ rank test, respectively, indicating a weak correlation. The correlation improves significantly if we remove one outlier, RXC J2157.4-0747 (the two component cluster, visible in the top left in both panels of Fig. 2). In this case the corresponding probability for no correlation decrease to 0.22 and 0.12 , giving stronger significance to the correlation. We also studied how the correlation changes for power ratios determined with smaller apertures and found a significant improvement in the correlation statistics for aperture sizes of $0.7-0.8 R_{500}$, as listed in Table 3 and as further discussed in the Appendix.

\section{Observations versus simulations}

\subsection{Dependence on viewing angle in simulations}

The simulations provide us with the means for another important approach to test the significance of the methods for the characterisation of substructure. Since for the 121 simulated clusters, images from three different orthogonal viewing angles are at hand, we can test how much the substructure characterisation varies depending on viewing angle. Therefore we can straightforwardly investigate how well the structure parameter results are correlated for the three different projections of each cluster. Figure 4 shows the results for two projections of the power ratio $P_{3} / P_{0}$. There is a clear correlation and also a very large scatter.

Similarly we compare the correlation of the w-parameter determined for different viewing angles in Fig. 5. The correlation

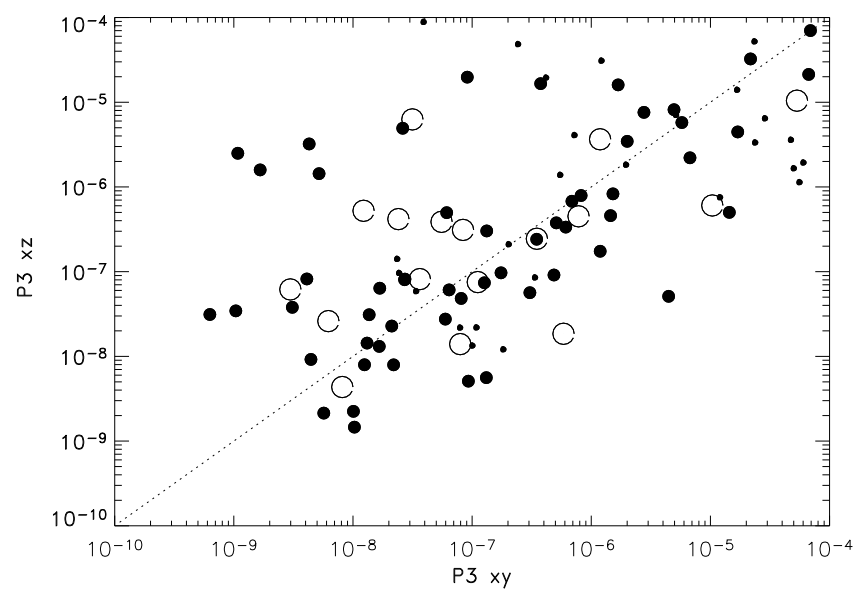

Fig. 4. Comparison of the $P_{3} / P_{0}$ result for two different orthogonal projections of the simulated clusters, showing a clear correlation with a large scatter. The larger points mark clusters with temperatures above $2 \mathrm{keV}$, the open symbols those with $T_{\mathrm{X}}$ above $3.5 \mathrm{keV}$. The dashed line indicates equality of both parameters.

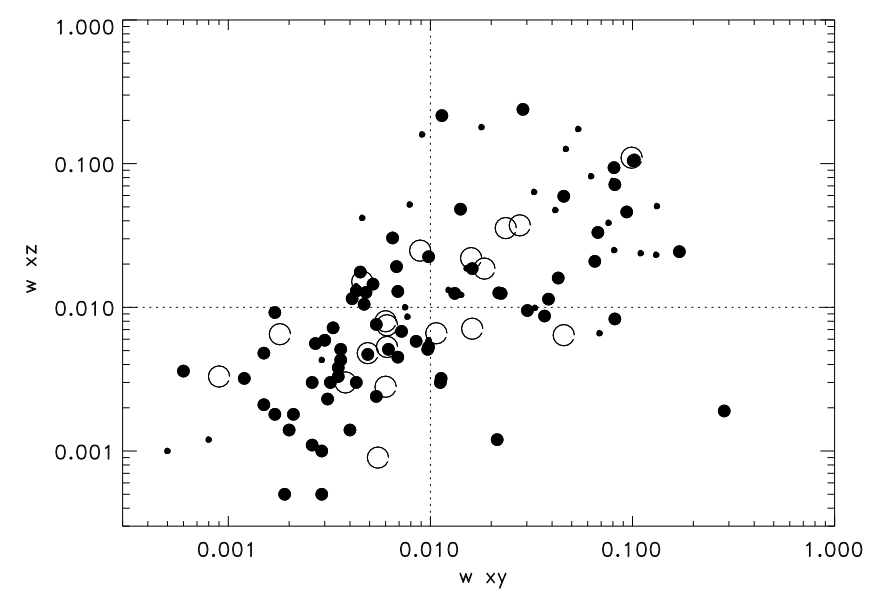

Fig. 5. Comparison of the centroid shift statistic parameter, $w$, for two different orthogonal projections of the simulated clusters, showing a clear correlation with a large scatter. The larger dots mark clusters with temperatures above $2 \mathrm{keV}$, the open symbols those with $T_{\mathrm{X}}$ above $3.5 \mathrm{keV}$. The dashed lines indicate the defined threshold for considering a cluster as dynamically distorted for both viewing directions.

as shown in the plot seems tighter than that for $P_{3} / P_{0}$. We have determined the mean orthogonal scatter (standard deviation from the diagonal) of the power ratios and the $w$ parameter for all three projection pairs, averaging using both linear and logarithmic summation. The results are summarised in Table 4 . The standard deviation of the values is about as large as the values themselves; however, we note that the mean orthogonal scatter for $P_{3} / P_{0}$ is about twice as large as the scatter for the center shift parameter, $w$.

\subsection{Comparison of observations and simulations}

As mentioned earlier, the simulation images do not contain photon noise, so that the substructure parameters we obtain have no statistical error and we also do not subtract a photon noise bias. Figure 6 shows a comparison of the distribution of $P_{2} / P_{0}$ and $P_{3} / P_{0}$ for the simulations and observations. Since the simulation sample contains a large number of low temperature clusters outside the selection interval of REXCESS we have marked 
Table 4. Comparison of the mean orthogonal scatter of the substructure parameters from the analysis of the simulated clusters seen from different projection angles.

\begin{tabular}{lcccc}
\hline \hline Parameter pair & $\mathrm{X}-\mathrm{Y}$ & $\mathrm{X}-\mathrm{Z}$ & $\mathrm{Y}-\mathrm{Z}$ & Mean \\
\hline$\left\langle\Delta \widetilde{P_{2}} / \widetilde{P_{2}}\right\rangle / \sqrt{2}$ & 0.72 & 0.79 & 0.77 & 0.76 \\
$\exp \left\langle\ln \left(\Delta \widetilde{P_{2}} / \widetilde{P_{2}}\right)\right\rangle / \sqrt{2}$ & 0.48 & 0.56 & 0.57 & 0.54 \\
$\left\langle\Delta \widetilde{P_{3}} / \widetilde{P_{3}}\right\rangle / \sqrt{2}$ & 0.99 & 1.85 & 0.91 & 1.25 \\
$\exp \left\langle\ln \left(\Delta \widetilde{P_{3}} / \widetilde{P_{3}}\right)\right\rangle / \sqrt{2}$ & 0.63 & 0.83 & 0.72 & 0.73 \\
$\left\langle\Delta \widetilde{P_{4}} / \widetilde{P_{4}}\right\rangle / \sqrt{2}$ & 1.13 & 1.03 & 1.01 & 1.06 \\
$\exp \left\langle\ln \left(\Delta \widetilde{P_{4}} / \widetilde{P_{4}}\right)\right\rangle / \sqrt{2}$ & 0.71 & 0.80 & 0.80 & 0.77 \\
$\langle\Delta w / w\rangle / \sqrt{2}$ & 0.50 & 0.49 & 0.48 & 0.49 \\
$\exp \langle(\ln (\Delta w / w))\rangle / \sqrt{2}$ & 0.32 & 0.29 & 0.26 & 0.29 \\
\hline
\end{tabular}

Notes. $\widetilde{P_{2}}=P_{2} / P_{0}$, etc. The orthogonal scatter is defined as the mean deviation from the diagonal in the plot and thus the algebraic expressions in the table contain an extra factor of $1 / \sqrt{2}$. The mean is determined by both linear and logarithmic averaging. The means for the three projections and the total mean are given. The standard deviation of these parameters from the mean is slightly smaller than the means, but of the same order of magnitude.

the clusters with temperatures above $2 \mathrm{keV}$ with larger symbols. There is no apparent difference in the parameter distribution of the simulated clusters at $T_{\mathrm{X}} \leq 2 \mathrm{keV}$ and $T_{\mathrm{X}} \geq 2 \mathrm{keV}$. While a larger fraction of the simulated clusters cover a similar parameter space to the observed objects in $P_{2} / P_{0}$ and $P_{3} / P_{0}$, there is a substantial fraction of simulated clusters with much higher substructure measures than REXCESS. To show this more quantitatively we have determined the log-mean of the different distributions, as shown in the Fig. 6. The log-mean of the observed clusters is at much lower values in both parameters. We also show the logmean parameter value for the simulated galaxy clusters selecting only those systems with $T_{\mathrm{X}} \geq 2 \mathrm{keV}$, finding that the result does not differ significantly from that of the total sample. We have also checked that there is no significant difference using only clusters at $T_{\mathrm{X}} \geq 4 \mathrm{keV}$. The result that the substructure measures are largely independent of the cluster temperature when applied to the simulations supports the view that the discrepancy in mean values between the simulations and observations is not due to a mass or ICM temperature selection effect. We further corroborate these results with the analysis discussed below and shown in Fig. 15. Figure 7 shows the histograms of all the power ratio values for the observed and simulated systems, underlining the fact that the power ratios of the simulated systems extend to much higher values than the observed objects. Figure 8 shows the histograms of the $w$ parameter determined with and without excision of the centre. The discrepancy is more subtle in $w$ than for the power ratios, but is still significant.

Possible selection effects due to the different temperature and mass ranges covered by the simulated and observed cluster samples remains a major concern (e.g. 90 per cent of the simulated clusters have a temperature below $4.3 \mathrm{keV}$ but only 55 per cent of the observed clusters have temperatures below this value). We thus performed another test to show that the excess of simulated clusters with strong indications for substructure is not due to selection effects. We resampled the simulated clusters in such a way that the distribution in temperature is roughly similar to the observed distribution. The resampling is not exactly perfect, since we have only 3 clusters with 3 viewing angles each in the temperature range from 4.3 to $6.5 \mathrm{keV}$ and so we restore the balance by having more objects in the neighbouring bins. In total we compare 54 resampled clusters (treating the different viewing
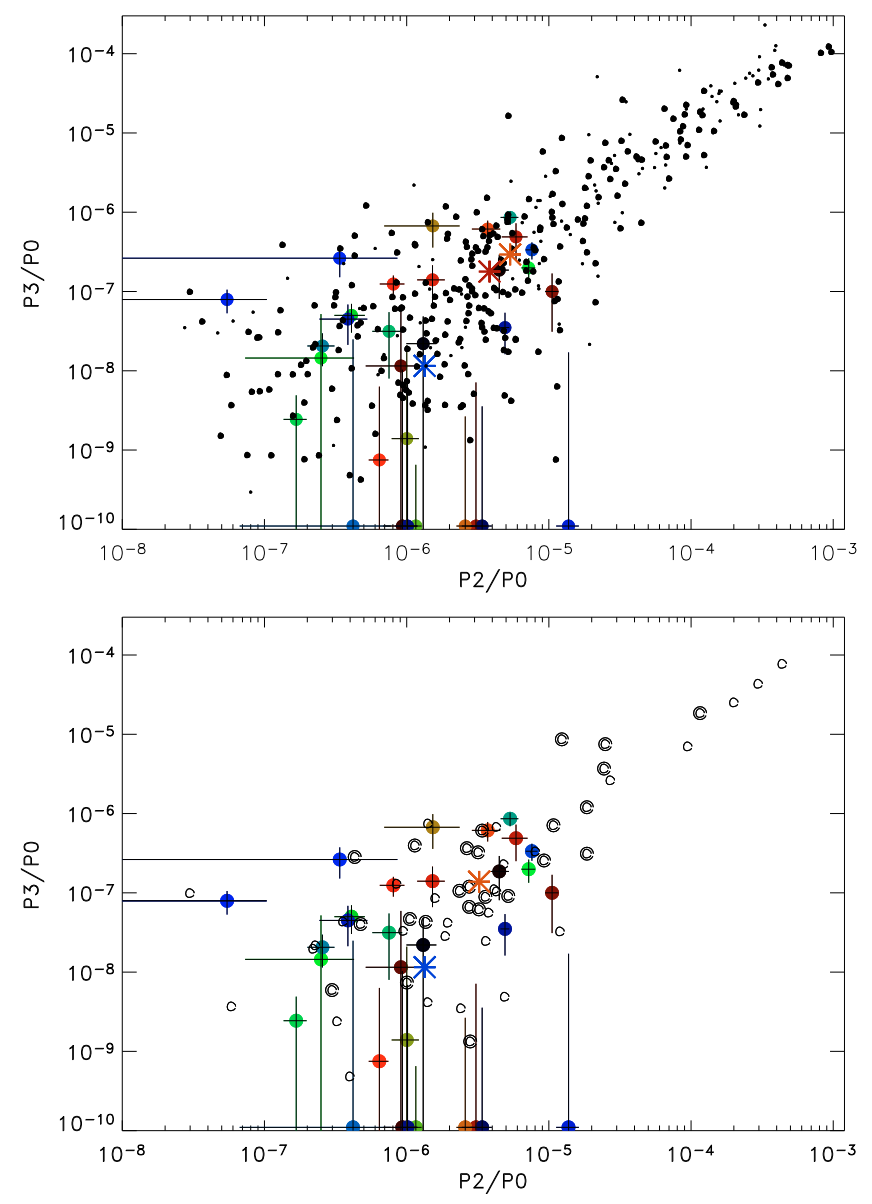

Fig. 6. Upper panel: comparison of power ratio parameter distributions of $P_{2} / P_{0}$ and $P_{3} / P_{0}$ for the observed and simulated clusters. The lower, blue cross indicates the log mean of the observed values and the upper crosses the values for the simulated clusters. Of these, the upper orange symbol is for all clusters and the slightly lower dark red mark for all simulated clusters with temperatures above $2 \mathrm{keV}$. Lower panel: same plot but now only a subsample of 54 clusters from the simulations is shown that has a very similar ICM temperature distribution as the observed clusters. Clusters with temperatures above $4 \mathrm{keV}$ are marked with larger double circles. In both plots we treat simulated galaxy clusters seen from different orthogonal viewing angles as independent objects.

angles of the same cluster as independent values) to the $31 \mathrm{ob}-$ served clusters with very similar temperature distributions in the lower panel of Fig. 6. We note that 22 per cent (12/54) of the simulated clusters have power ratios in excess of the regime covered by the observed clusters. We also use different symbols for simulated clusters below and above $4 \mathrm{keV}$ and note that, even given the small number, hotter and cooler clusters have similar power ratio distributions. Therefore we are confident that we can rule out that the discrepancy in the power ratio distributions between observed and simulated clusters is due to a selection effect.

A very similar result for the distribution of the power ratio parameters is found when comparing with the simulations by Valdarnini (2006). The $P_{3} / P_{0}$ of his simulated clusters span the range of $10^{-8}$ to $10^{-4}$, and thus these simulations also populate the parameter range from $10^{-6}$ to $10^{-4}$, in which there are no observed clusters. Earlier comparison of simulations and observations by Buote \& Xu (1997) and Valdarnini et al. (1999), which have been conducted to test the diagnostics of cluster morphology statistics to distinguish between different cosmological models, do not show such a large discrepancy (although 

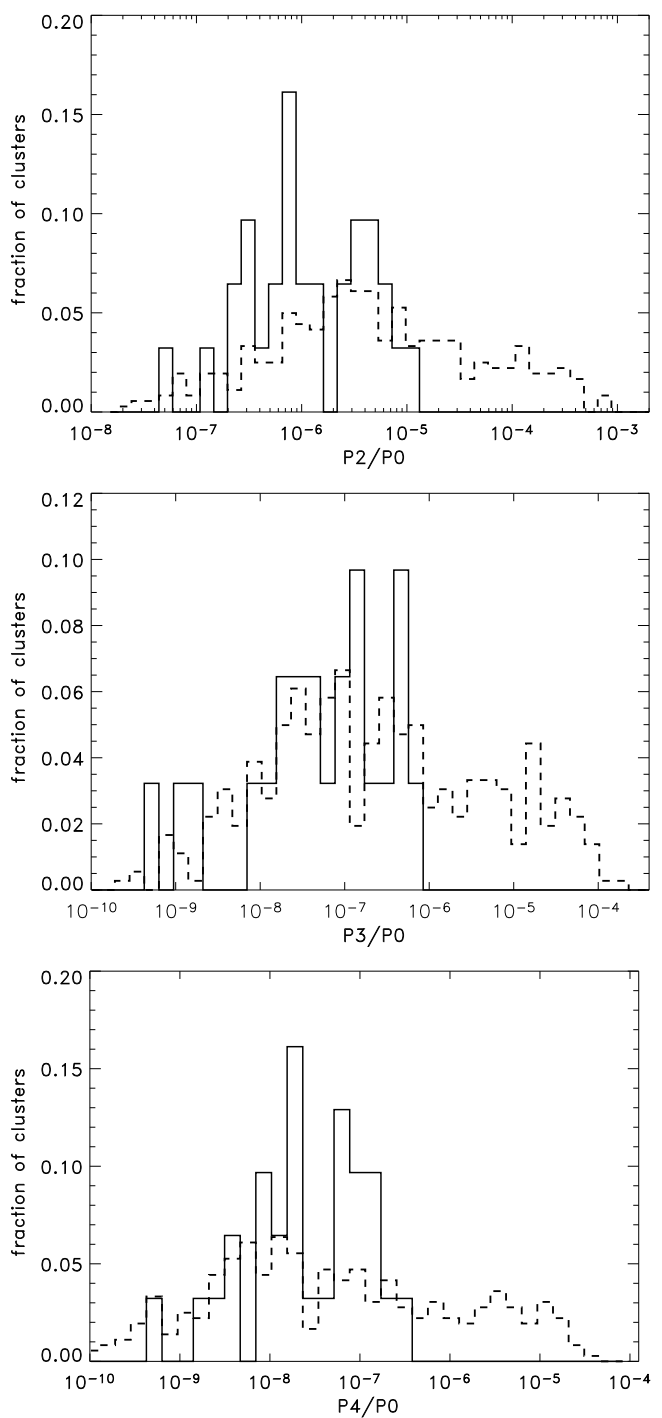

Fig. 7. Comparison of the distribution functions of, from top to bottom, $P_{2} / P_{0}, P_{3} / P_{0}$ and $P_{4} / P_{0}$, for the simulations (dashed lines) and observations (solid line). All histograms are normalised by the total number of clusters of each sample.

no good match was found with $\Lambda$ CDM cosmology). The simulations used there are only based on dark matter dynamics in the case of Buote and $\mathrm{Xu}$ and on adiabatic hydrodynamics in the case of Valdarnini et al., however.

In search of a physical reason for the difference in morphological statistics between simulated and observed clusters, we inspected the images of the simulated objects with large substructure parameters. Figure 9 shows four examples of simulated clusters from the extreme upper right corner of Fig. 6. These images contain only the diffuse X-ray emission of the ICM, and what may appear to be point sources are very compact cool regions that have been accreted by the clusters. We have marked in the figure the aperture radius $R_{500}$ inside which the substructure analysis is undertaken. All of the simulated clusters show noticeable substructure features inside $R_{500}$ that serve to boost the power ratios, in particular if they are located close to the aperture radius. In the real cluster images we do not find equivalent compact emission regions. It thus appears that one significant difference between observations and simulations is the fact that at least a fraction of the simulated clusters contain more compact cool cores than their observed counterparts.
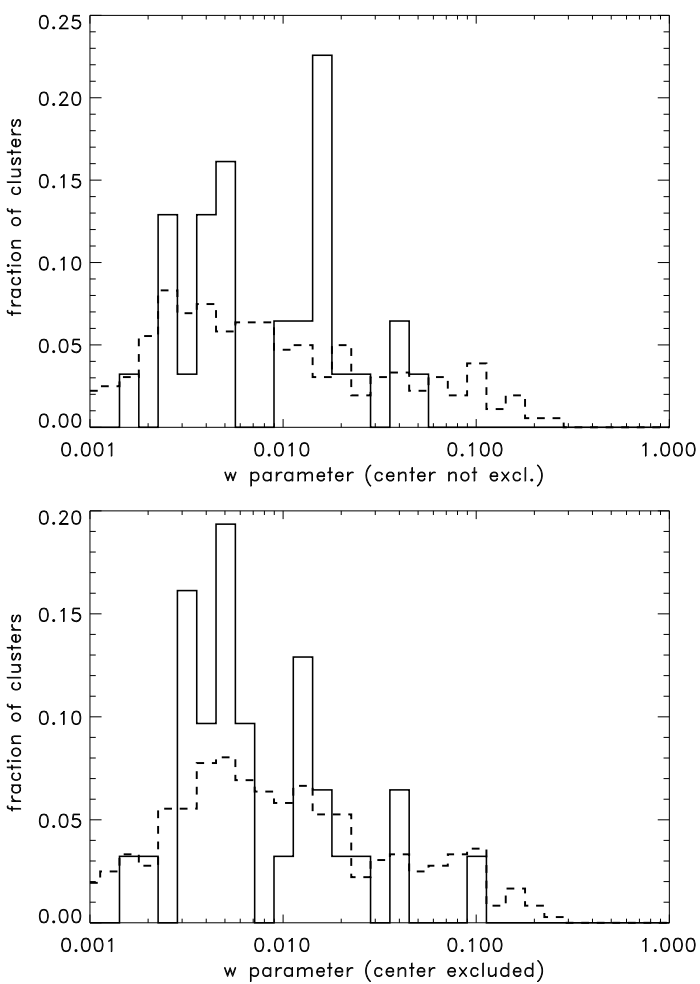

Fig. 8. Comparison of the distribution functions for the $w$-parameter obtained from the full aperture (top) and with central region excluded (bottom), for the simulations (dashed lines) and observations (solid line). Both histograms are normalised by the total number of clusters in each sample.

Pratt et al. (2007), when comparing the temperature profiles of the REXCESS with those from this same sample of simulated clusters, showed that almost all simulated objects had a central temperature decrease signifying the presence of a pronounced central cool core, whereas less than half of the observations showed this feature. From this finding one could have expected that simulated clusters are more regular on average, since cool core clusters have statistically less substructure than non-cool core clusters. The explanation is more complex. The simulated clusters not only have more pronounced cool cores in their central regions, but they also contain previously accreted subclusters that themselves have strong cool cores. These survive in the final cluster and produce multiple maxima, as seen in Fig. 9. We can illustrate the overabundance of cool regions in the simulations with another statistic from our analysis. If we characterize the strength of a cool core by $L_{\text {rat }}$, the ratio of the cluster flux from the total cluster image interior to $R_{500}$ to that with the core region $\left(r<0.1 R_{500}\right)$ excised, we find, as shown in the histogram in Fig. 10, that the simulations cover a somewhat broader range of such flux ratios, extending up to higher values than the observations.

\section{Correlation of morphological and global cluster parameters}

In this section, we investigate how the substructure measures vary with global cluster properties. We start by investigating how the substructure parameters vary with mass, since this is the most fundamental scaling parameter of a cluster. We use the REXCESS mass estimates given in Pratt et al. (2010), which were obtained from iteration about the $M_{500}-Y_{\mathrm{X}}$ relation. 

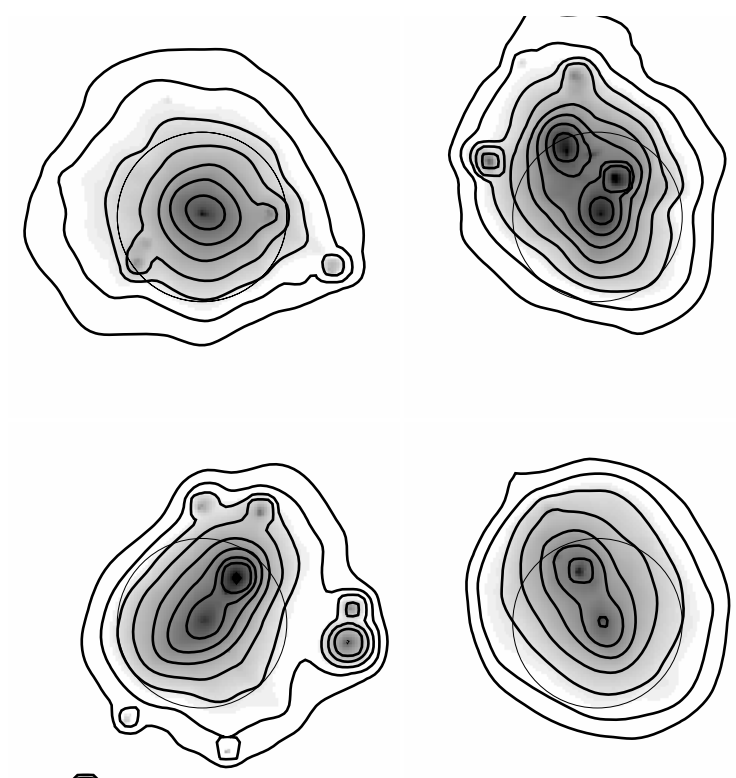

0

Fig. 9. Examples of the four simulated clusters from Borgani et al. (2004) having the largest values of $P_{3} / P_{0}>10^{-4}$. The thin circle indicates a radius of $R_{500}$. All of these clusters show several clear maxima or clumps inside $R_{500}$, in contrast to the images of the REXCESS clusters. The images show only the diffuse emission from the ICM and the compact emission regions are not point sources but are in fact small cool cores.

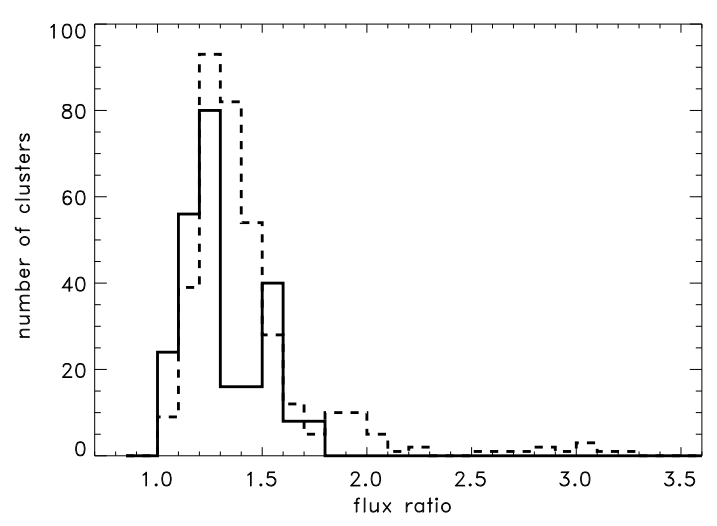

Fig. 10. Comparison of the ratio of the total cluster flux to the flux with the core region $\left(r<0.1 R_{500}\right)$ excised for 31 clusters from REXCESS (solid line) and the simulated clusters (dashed line).

Figure 11 shows $w$ and $P_{3} / P_{0}$, obtained with central region excised, as a function of mass. Also overplotted are logarithmically averaged values in three mass bins. There is no obvious variation in the occurrence and strength of substructure with cluster mass, a result that is quantitatively confirmed by the statistical tests listed in Table 3. For $w$, a Kendall's $\tau$ test gives a probability of 0.81 and a Spearman's rank test a probability of 0.81 for no correlation. For $P_{3} / P_{0}$, the corresponding probabilities are 0.69 and 0.73 , clearly pointing towards no mass correlation.

Next we use bolometric X-ray luminosity, $L_{X}$, as the scaling parameter, since this is the most frequently used observable. Figure 12 shows $w$ and $P_{3} / P_{0}$ as a function of the REXCESS $L_{X}$ values published by Pratt et al. (2009), with averages in three $L_{X}$ bins overplotted. The Kendall's $\tau$ and Spearman's $\rho$ probabilities are $0.63(0.14)$ and $0.63(0.22)$ for $w\left(P_{3} / P_{0}\right)$, respectively, suggesting that at least for the correlation of the power ratios
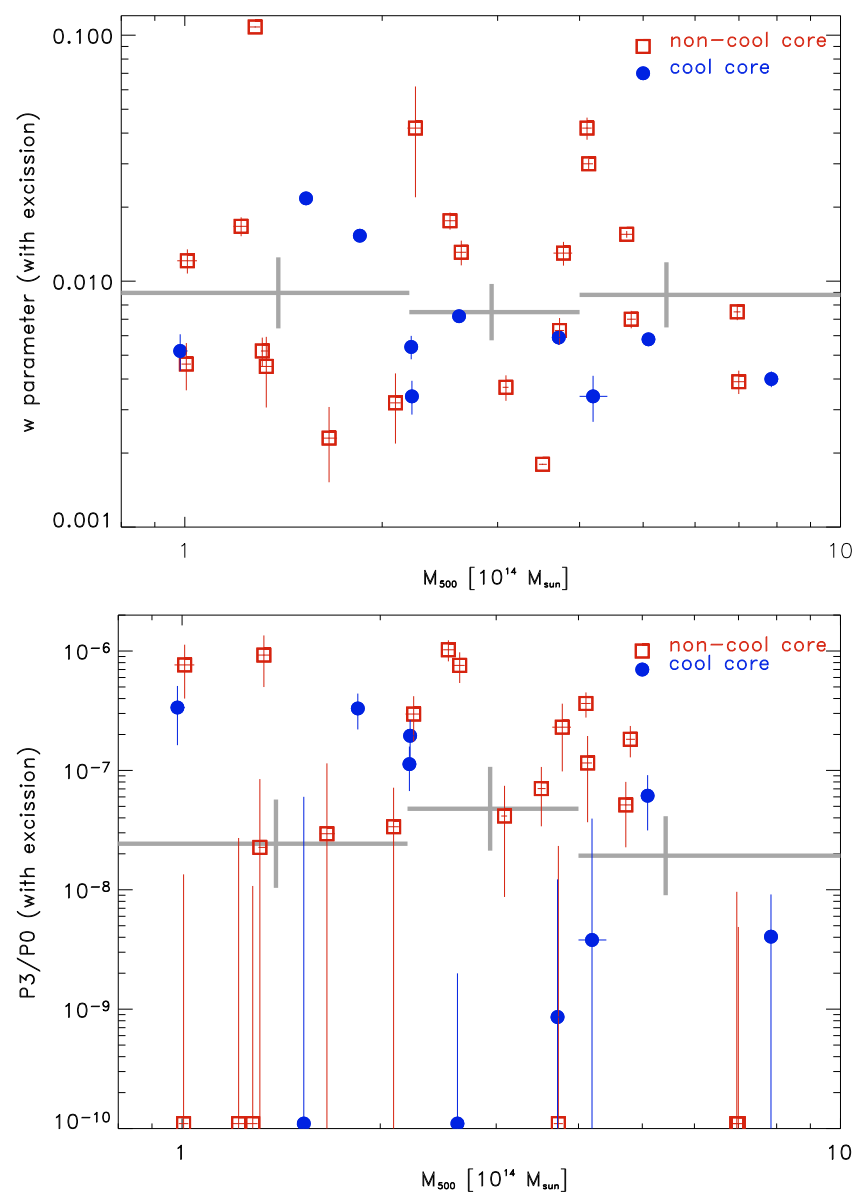

Fig. 11. Correlation of the substructure parameter $w(t o p)$ and $P_{3} / P_{0}$ (bottom) with cluster mass for the 31 cluster from REXCESS. The grey bars show the log-mean values of $w$ and $P_{3} / P_{0}$ determined for three mass bins.

with X-ray luminosity the observed weak correlation is statistically confirmed.

We further investigate how substructure and the cool core properties are connected. This was already partly explored in Croston et al. (2008, their Fig. 12) using correlations of $w$ and $P 3 / P 0$ with central gas density (at $0.007 R_{500}$ ) and central cooling time (at $0.03 R_{500}$ ). Croston et al. found that these data allowed to reject the hypothesis of no correlation with probabilities of $\sim 85-92 \%$. We show a similar analysis for the power ratios in Fig. 13. A Kendall's $\tau$ test gives a probability of no correlation of $12 \%(10 \%)$ and a Spearman rank test a probability of $6 \%(6 \%)$ for central density (cooling time), respectively. We have also studied the variation of $w$ with the central density and cooling time - results are given in Table 3 . The correlation with $w$ is even tighter than for $P_{3} / P_{0}$. In a study presented in Sect. A.3 in the Appendix, we show that also the strength of the correlation between $P_{3} / P_{0}$ and cool core indicators increases if we decrease the aperture radius, thus giving less weight to the very outer regions of the object.

There is thus evidence for a reasonably good correspondence between global morphological parameters and core properties. This quantifies for the first time in a representative sample the widely expected result that cool core systems correspond to clusters that have not been disturbed by mergers in the recent past. But the fact that the correlation is far from being perfect implies that the presence of a cool core can not generally be taken as an indication that a cluster is relaxed. On the other hand, it is worth 
H. Böhringer et al.: Substructure in REXCESS galaxy clusters
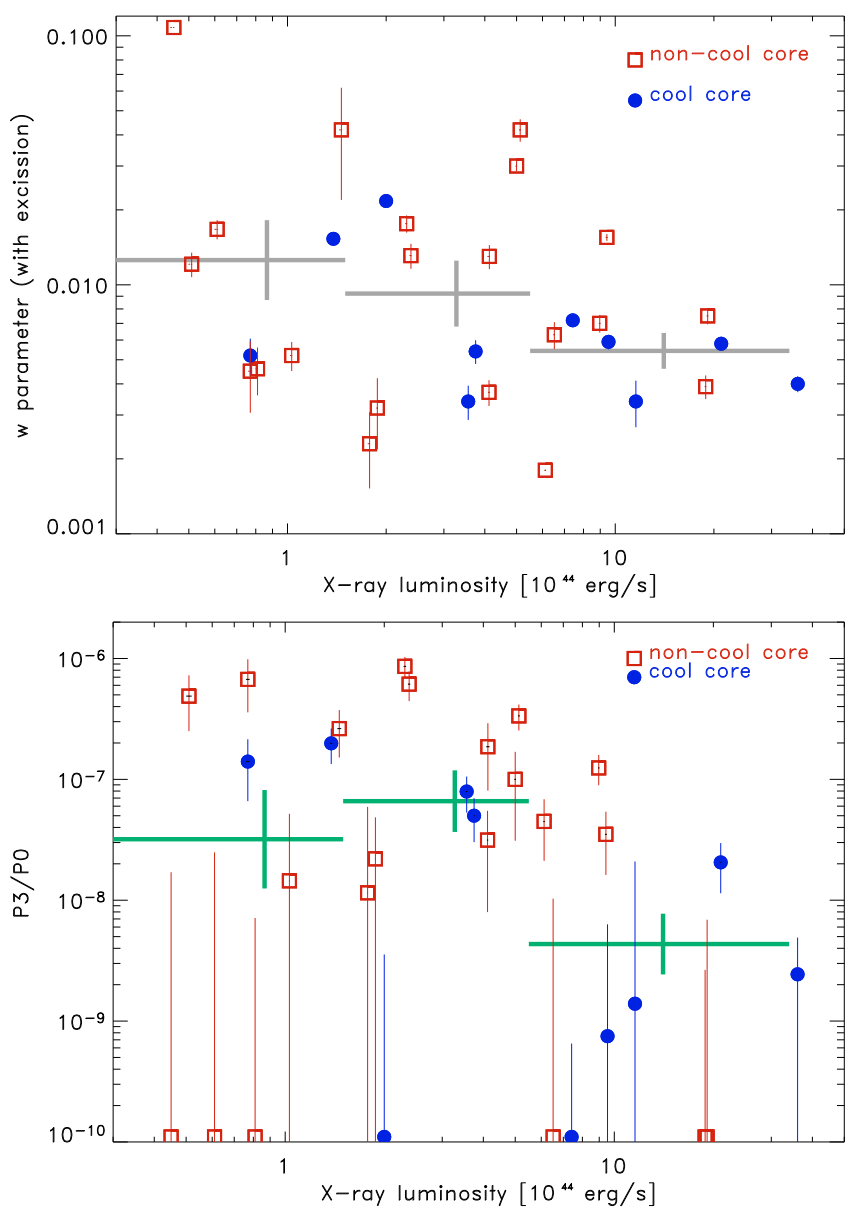

Fig. 12. Correlation of $w$ (top) and $P_{3}$ (bottom), both obtained with the core regions excised, with bolometric X-ray luminosity estimated interior to $R_{500}$ aperture (Pratt et al. 2009). The grey bars show the log-mean values of $w$ determined for three luminosity bins.

noting that the correlation seen in Fig. 13 is all the more remarkable given that it involves $P_{3} / P_{0}$, which provides a measure of substructure on a very global scale with little influence from the central regions, as discussed above. This is the reason why we preferred to show this relation rather than the tighter correlation with the $w$ parameter. The correlation we find therefore demonstrates that there is a causal, statistical connection between the properties of the very central region and the global morphology.

The above results provide the key to understanding the different correlations between mass, X-ray luminosity and cluster morphology. We know that for a given mass, clusters with cool cores have in general higher X-ray luminosities (e.g. Fabian et al. 1994; Chen et al. 2007; Pratt et al. 2009). Thus in going from the mass distribution to the $L_{X}$ distribution, cluster cool cores preferentially move to higher luminosities compared to non-cool core clusters. Since these cool core clusters are on average more regular, the more regular clusters will accumulate at the higher luminosity side - exactly as observed. Thus, if we accept galaxy cluster mass as the primary scaling parameter, the correlation of $L_{\mathrm{X}}$ with the substructure parameters can be seen as a selection effect.

To close the loop of arguments, we can also test our expectation that cool cores are preferentially found in the higher luminosity bins. Figure 14 shows the luminosity ratio $L_{\mathrm{rat}}$, defined as the ratio of the total flux in the $[0.5-2] \mathrm{keV}$ band measured interior to $R_{500}$ to the flux in the same aperture with the core
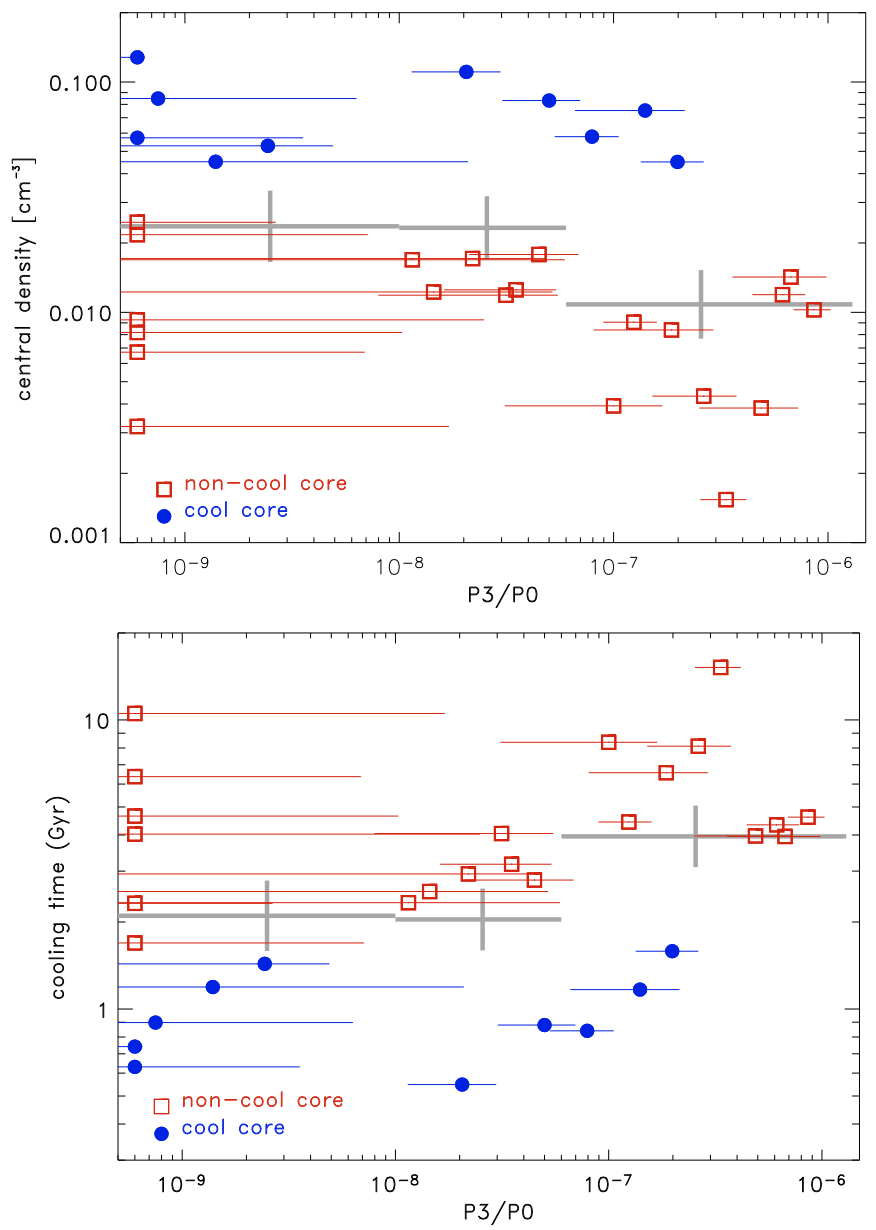

Fig. 13. Correlation of the central ICM density (top) and cooling time (bottom) with the $P_{3} / P_{0}$. Similar correlations between the centre shift parameter $w$ and the central density and cooling time were shown in Fig. 12 of Croston et al. (2008).

region $\left(r<0.1 R_{500}\right)$ excised. This parameter was defined to separate out cooling cores and is, as we have tested, very tightly correlated to central density and cooling time. There is a noticeable correlation and it is mostly the highest luminosity bin that features a higher $L_{\mathrm{rat}}$ on average. The statistical tests listed in Table 3 show a probability of non correlation of 0.16 and 0.18 on a Kendall's $\tau$ test and a Spearman's rank test, respectively, supporting a significant correlation. If the same test is done for the correlation of $L_{\text {rat }}$ with the cluster mass, as shown in the lower panel of Fig. 14, we find that it is rejected with probabilities of 0.97 and 0.84 on a Kendall's $\tau$ test and Spearman's rank test, respectively. In the middel panel of Fig. 14 we also show the correlation of $L_{\text {rat }}$ with the core excised X-ray luminosity of the clusters. The no-correlation probabilities of 0.97 and 0.84 provided by Kendall's $\tau$ test and Spearman's rank tests indicate no significant correlation. Thus, core excision in the luminosity integration removes the influence of cool cores quite effectively.

\section{Discussion}

\subsection{Methodology}

The results presented in this paper provide insight into the reliability and sensitivity of two methods to characterise substructure: power ratios and centre shifts. We have introduced new methods to estimate the bias produced by photon noise and to 

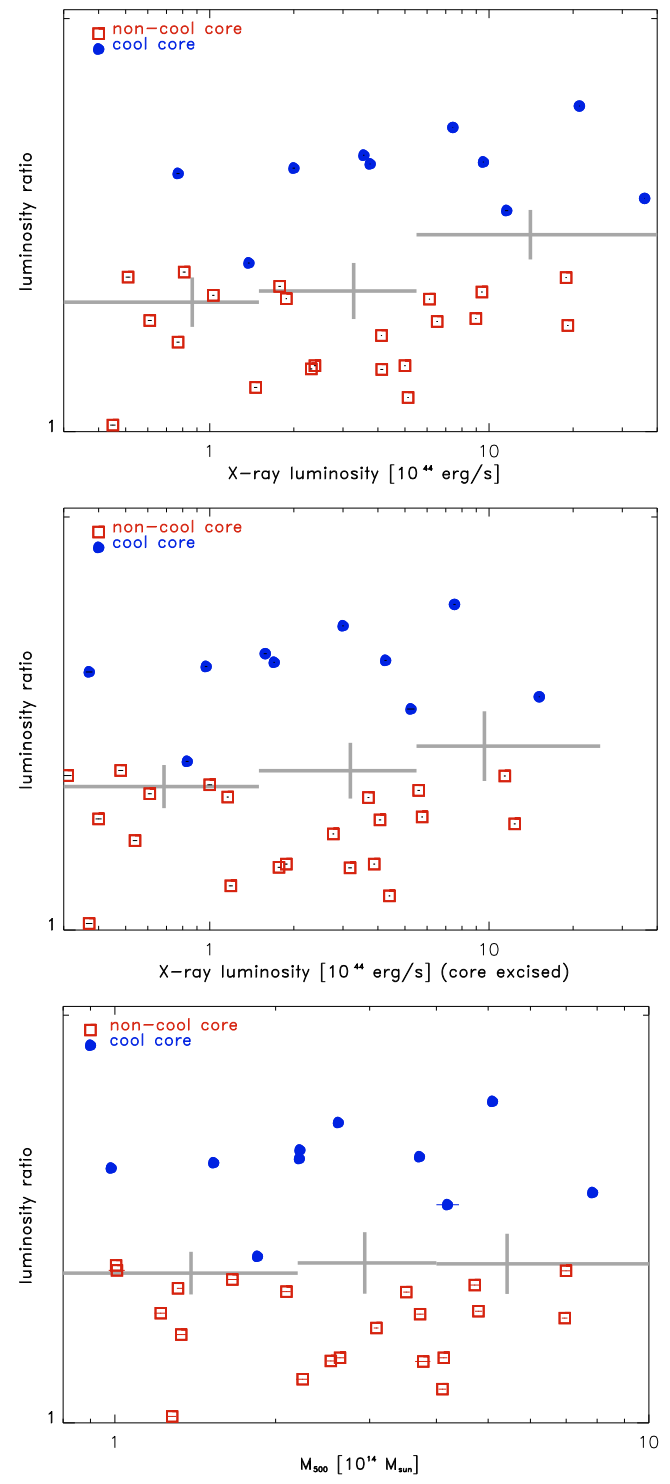

Fig. 14. Correlation of the bolometric X-ray luminosity interior to $R_{500}$ (top), core excised bolometric X-ray luminosity (middle), and mass (bottom) with the X-ray luminosity ratio $L_{\text {rat }}$. The latter is defined as the ratio of the total flux in the $[0.5-2] \mathrm{keV}$ band measured interior to $R_{500}$ to the flux in the same aperture with the core region $\left(r<0.1 R_{500}\right)$ excised. This luminosity ratio closely correlates with other cool core properties such as central density and cooling time, and is a sensitive indicator of cool cores. The grey bars show the log-mean values of $L_{\mathrm{rat}}$ determined for three bins.

assess the uncertainties in the results. Our analysis suggests that, while these morphological characterisations are not precision measures for individual clusters, they can still provide useful statistics to study trends of properties in samples of clusters. This is evident from both the results of the Monte Carlo Poisson noise simulations to estimate uncertainties, and from the difference in the substructure measures of simulated clusters when obtained from different viewing angles.

In a comparison of the morphological parameters $P_{3} / P_{0}$ and $w$, we find typical uncertainties (for good XMM-Newton data quality with high photon statistics) of $\sim 70$ per cent and $\sim 15$ per cent, for power ratios and $w$, respectively. Testing the recovery of a given substructure measure from the observation of a (simulated) cluster from different viewing angles, we find that the difference for the different viewing angles is about as large as the values of $P_{3} / P_{0}$ itself, while it is smaller by about a factor of two for $w$. Yang et al. (2008) have recently found similar results using power ratios and centroid shift tests on simulated clusters with known merger histories. They find a substantial and significant correlation of $P_{2} / P_{0}, P_{3} / P_{0}$, and $w$ with the time passed since the last major merger (for a mass ratio smaller than 5:1). Similar to our findings the correlation is not tight enough for a cluster by cluster identification of the dynamical state, but it provides important statistical diagnostics. In addition they find that $w$ is significantly more sensitive than the power ratios. For our work with the REXCESS sample we have therefore adopted a threshold value of $w>0.01$ for the designation of a galaxy cluster as being dynamically disturbed.

Despite the fact that $w$ is a more sensitive substructure diagnostic, one should not easily conclude that power ratios should be given up as an alternative. For multi-peaked (simulated) clusters, the power ratios pick up the obvious substructure with higher sensitivity than the centroid shifts, as can be seen in the larger relative excesses of the $P_{3} / P_{0}$ values compared to the $w$ values in Figs. 7 and 8, respectively. For the substructure - cool core correlation $w$ shows a much stronger connection (likely because it is less biased toward the signal from the outer radii), but it is $P_{3} / P_{0}$ that is more affected by the correlation with $\mathrm{X}$-ray luminosity (Fig. 12). This illustrates that the different methods of characterising substructure react differently to various morphologies and it may still be useful to look at several substructure tests in cluster morphology studies.

Our results also point towards a possible future improvement in our application of the power ratio method. Firstly, as shown in the Appendix, running the power ratio analysis in several apertures tends to emphasise different structural features. Furthermore, we have several cases of highly disturbed clusters in our sample, where we see large quadrupole and/or octopole moments but no significant hexapole signal, because the distortion preserves some mirror symmetry. This suggests that it might be worthwhile to investigate a composite power ratio measure that combines the several multipole moments at various radii. This idea can be seen in analogy to the definition of the centre shift parameter, which is also derived from statistics of measurements with several apertures.

Finally we reemphasise that the clear statistical difference between the cool core versus non-cool core clusters, plus that of observed versus simulated clusters, provides a nice illustration of the power of the substructure measures as a statistical diagnostic of the sample under consideration.

\subsection{Relations between cluster properties}

One of the most interesting findings of this study is that there is no mass dependence of the substructure statistics. This is not only revealed by the observed cluster sample, which might still be affected by small number statistics, but is also shown by the simulated cluster sample, as shown in Fig. 15. Naively one might expect in the standard cosmological model of hierarchical structure formation, where the largest structure are the youngest, that larger clusters have had more recent mergers and therefore show on average larger substructure measures. However, some theoretical studies show that this might in fact be a very mild effect. Guo (2009) has for example studied the growth of dark matter halos via major mergers (defined by mass ratios less than three) in the Millenium simulations (Springel et al. 2005), and finds that the merger rate differs by less than 20 per cent for mass differences of a factor of four in the mass range relevant for our 


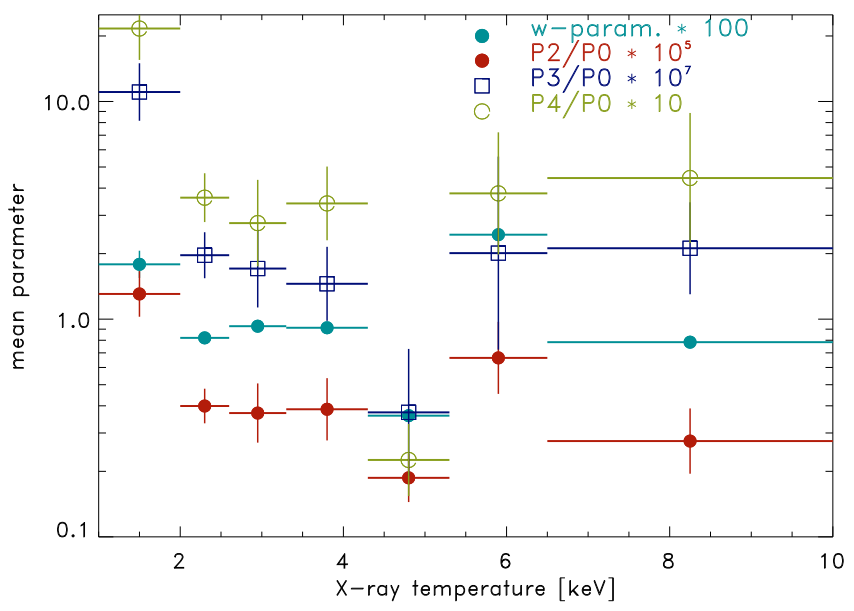

Fig. 15. Substructure measures, power ratios and centershifts, for the simulated clusters as a function of ICM temperature. The substructure measures have been averaged for seven temperature bins of the simulated cluster sample. For each cluster all three projections have been used as independent results. Note that bin 5 contains only one and bin 6 only two clusters.

sample. This indicates that our finding may be well consistent with the currently adopted structure formation scenario.

The independence of cluster mass and morphology in our sample is then also reflected by the fact that there is no mass bin which has preferentially more cool cores. This is slightly different from the result of an analysis of the 106 brightest known galaxy clusters (the HIFLUCGS sample), where a bias towards more cool core clusters in low mass systems was found (Chen et al. 2007).

The very low correlation of substructure with cluster mass is good news for the application of galaxy clusters to cosmology. One of the most critical tasks in these cosmological studies is the construction of robust relations between simple cluster observables and cluster mass. The most problematic issue here is that observed cluster samples consist of a mixture of relaxed and unrelaxed clusters. In this context it is of great help for the construction and calibration of observable - mass relations to know that the ratio of relaxed to unrelaxed clusters is not a strong function of mass.

In studying the correlation of cool core properties with substructure this paper adds a new dimension to previous work in two respects: (i) by using a morphologically unbiased sample with selection purely by X-ray luminosity, and (ii) by analysing cluster properties out to a fiducial global radius, $R_{500}$. Cool core objects unsurprisingly show a very regular appearance in low exposure images or in images obtained with earlier X-ray observatories, since in such exposures we mainly detect the bright centre, which is always very regular. In contrast to this, our study involves the cluster appearance out to a large radius, and we have carefully examined the effect of cool cores by providing results where the cool core has been excised. This provides a new look at some facets of the cool core cluster morphology relation problem. For example, in the present sample, we do indeed find globally disturbed clusters which harbour cool cores.

\subsection{Observations versus simulations}

The comparison of observations and simulations in Sect. 4.4 provides significant evidence that the physical recipes used to model the evolution of the intracluster medium by cooling, feedback and diffusive transport processes differs from the processes prevailing in nature. Since the prime goal of the simulations was to reproduce the observed scaling relations of the various global ICM properties, cluster morphological parameters have rarely been used to tune the simulation recipes. One easily-identified difference between the simulations and the observations is the presence of more pronounced cool regions in the simulated clusters, as explained above. This was also seen in Pratt et al. (2007), where almost all simulated clusters feature central temperature drops, whereas only about a third of the observed clusters have cool cores.

As a consequence of the pronounced cool cores in the simulated objects, we find merger remnants with two or more cool cores which are not yet or incompletely disrupted. One of the REXCESS clusters excluded from the present analysis, RXC J2152.2-1942, is a double cluster, but the two cluster centers are well separated and lie outside each others $R_{500}$, which distinguishes this cluster from the simulation examples shown in Fig. 9. Similarly, the other excluded cluster RXC J0956.4-1004 (the A901/902 supercluster) shows three well-separated X-ray emission regions.

The simulations used here were performed in 2004. Since then new recipes have been introduced to cosmological cluster simulations, including a significant feedback from central AGN to partly suppress the formation of cool cores. This has recently been explored both semi-analytically (e.g. Croton et al. 2006; Bower et al. 2006), and also in $N$-body/hydrodynamical simulations (e.g. Sijacki et al. 2008; Puchwein et al. 2009; Fabjan et al. 2009). It will be one of our next projects to extend the comparison to this new generation of simulations.

The lower degree of substructure seen in observations, as compared to simulations is advantageous for the cosmological application of galaxy clusters. Some of the problems pointed out in simulation studies using numerical prescriptions similar to the simulations used here, such as e.g. the effect of multitemperature structure on the determination of cluster masses from X-ray observations (Mazzotta et al. 2004; Rasia et al. 2005, 2006) should be less problematic if extra-central cool core regions in clusters are rare in nature, and the temperature distribution in clusters is in general more regular. This highlights the importance of testing the compatibility of the simulated clusters with representative samples of observed objects using all possible observational characteristics, to ensure their physical similarity.

\section{Conclusions}

We have used power ratios and centre shifts to investigate the substructure and morphological characteristics of 31 clusters from the REXCESS galaxy cluster sample. We examine in parallel a sample of 117 clusters identified from hydrodynamical simulations of a $\Lambda \mathrm{CDM}$ model. Substructure measures are estimated consistently within a radius of $R_{500}$. Our main conclusions are as follows:

- Using a newly-developed Monte Carlo procedure to estimate the uncertainties, we find that $w$ is more sensitive than power ratios for the good quality cluster images we have at our disposal, although combination of the two methods gives complementary information.

- Neither substructure measure gives an exact quantification of a cluster's dynamical state, and so they should only be used in a statistical sense. 
- For both observed and simulated cluster samples, the substructure parameters do not exhibit a mass dependence, a result that has important implications for the construction and calibration of the observable-mass relations for use in cosmological applications.

- Cool core objects are generally the most regular. However there exist cool core systems that are identified as disturbed using both $w$ and power ratio substructure statistics.

- As compared to the observations, the simulations contain many more cool, dense regions. This contributes to a statistical enhancement in the amount of substructure in the simulated clusters as compared to the observed objects, indicating that numerical prescriptions do not precisely reproduce the structure of the real cluster population.

Finally, we re-emphasise that in the present work we could only obtain statistically meaningful results because (i) we deal with a statistically representative sample, (ii) we have good photon statistics from deep XMM-Newton observations of relatively bright, not too distant clusters, and (iii) the data quality of the observed sample is fairly homogeneous. Any deviation from these ideal conditions would have made the analysis more difficult and less reliable.

Acknowledgements. The paper is based on observations obtained with $X M M-N e w t o n$, an ESA science mission with instruments and contributions directly funded by ESA Member States and the USA (NASA). The XMM-Newton project is supported in Germany by the Bundesministerium für Bildung und Forschung, Deutsches Zentrum für Luft und Raumfahrt (BMBF/DLR), the MaxPlanck Society and the Haidenhain-Stiftung. G.W.P. acknowledges partial support from DfG Transregio Programme TR33. H.B. acknowledges support for the research group through The Cluster of Excellence "Origin and Structure of the Universe", funded by the Excellence Initiative of the Federal Government of Germany, EXC project number 153. K.D. acknowledges support from the DfG Priority Programm SPP 1177.

\section{Appendix A: Further details of the data analysis}

In addition to Table 1 in the main text of the paper, we provide here the complementary Table A.1, where the substructure parameters are listed for the analysis where central regions were not excluded. For the power ratios the differences are in almost all cases not larger than the uncertainties. For the $w$ parameter the results are also similar, but due to the fact that this parameter is less biased towards large radii and the better precision with which this parameter can be determined, the differences are in some cases larger than the uncertainties.

\section{A.1. Bias and error estimation for the power ratio method}

In this Appendix, we explain the new methods we have used to estimate photon noise bias and substructure measure uncertainties. Having performed the power ratio analysis as outlined in Eqs. (1) to (4), the first question we should ask in interpreting the results is: when have we detected a significant signal of deviation from azimuthal symmetry? Even a completely symmetric cluster in nature would be detected with some residual structure due to the photon noise with which it is observed. To asses this we perform the following test. We conduct a second substructure analysis in which for all flux pixels entering the integrals of Eqs. (3) and (4) we take only the radius from the data but assign randomly drawn angles $\phi$. This randomises all azimuthal structure the cluster might have. We repeat this process 1000 times and determine the mean and dispersion of the distribution of the power ratio parameters. The mean of the signal can be interpreted as the typical residual signal a perfectly regular cluster

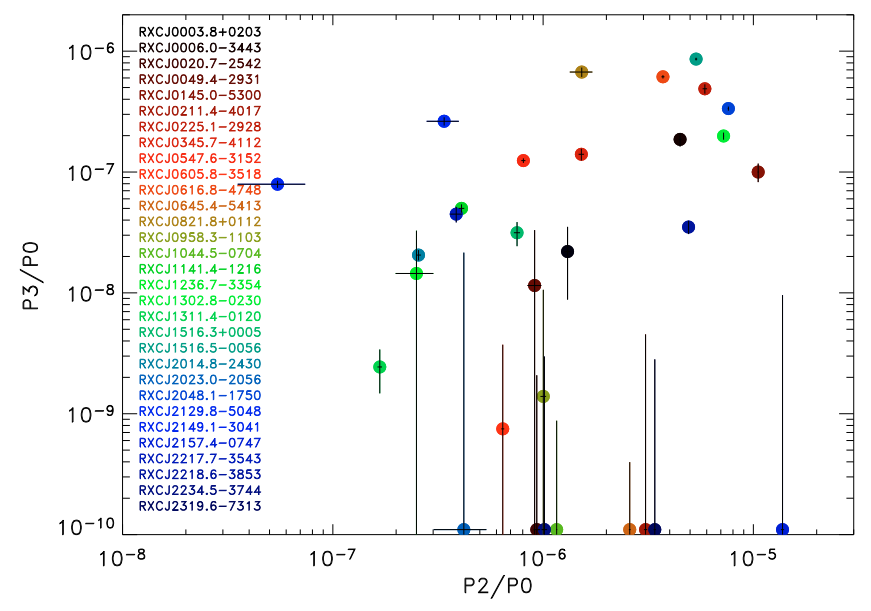

Fig. A.1. $P_{2} / P_{0}$ and $P_{3} / P_{0}$ for the 31 galaxy clusters of the REXCESS sample. The errors shown are those from simulations with azimuthal randomization used to test against the null hypothesis that the cluster is azimuthally symmetric. After correction for the signal contribution from photon noise, 8 clusters in the sample have negative values of $P 3 / P 0$ that are consistent with zero within the error limits. These data points are indicated at the bottom of the plot.

would have in the presence of photon noise. We interpret this spurious signal as a measure of the typical photon noise contribution to the power ratio measurement in all clusters and subtract its value from the obtained result to recover the intrinsic signal of the power ratio. The standard deviation of the spurious signal from the mean in all simulations is used as a first estimate of the uncertainty of the final net result. Some very regular clusters come out with a negative signal after subtraction of the photon noise contribution. But this negative signal is never larger than the uncertainty.

Figure A. 1 shows $P_{2} / P_{0}$ and $P_{3} / P_{0}$ for the REXCESS sample, with uncertainties estimated from the azimuthal randomisation process. The mean signal bias and associated uncertainties are always very similar, which is not surprising. Inspecting the uncertainties we note that for the data quality of the REXCESS sample we need typical values of $P_{2} / P_{0} \geq 10^{-7}, P_{3} / P_{0} \geq$ $2-4 \times 10^{-8}$ and $P_{4} / P_{0} \geq 2 \times 10^{-8}$ in order to be able to claim significant detection of substructure or deviations from azimuthal symmetry.

The above considerations provide a useful uncertainty estimate for clusters which have substructure signals close to the significance threshold. If a cluster has a strong substructural feature, the uncertainty of the measurement of the substructure parameter will depend critically on the photon noise connected to this feature, which is probably not related to the photon noise of a randomized cluster where this feature has been washed out. Therefore we need a new approach to estimate uncertainties. To test what uncertainties are expected for clusters with larger signals, we performed the following simulations. We used the combined count image (without background subtraction) and obtained a new integer random number for the photon counts in each pixel by drawing the numbers from a Poissonian distribution with the observed photon counts as expectation values. The Poissonized image is background and exposure corrected and subjected to precisely the same substructure analysis as the observed clusters. The process was repeated 200 times, and the mean and standard deviation determined. The mean simulated values are slightly higher than the observed values. This is due to the fact that the simulated images contain the artificial Poissonized noise on top of the observational noise, which 
Table A.1. Substructure parameters for 31 clusters from the REXCESS sample.

\begin{tabular}{|c|c|c|c|c|c|c|c|c|c|c|c|}
\hline Cluster & $\begin{array}{r}P 2 / P 0 \\
\times 10^{-5} \\
(2)\end{array}$ & $\begin{array}{r}\text { Bias } \\
\times 10^{-5} \\
(3)\end{array}$ & $\begin{array}{r}\text { Error } \\
\times 10^{-5} \\
(4)\end{array}$ & $\begin{array}{r}P 3 / P 0 \\
\times 10^{-7} \\
\quad(5)\end{array}$ & $\begin{array}{r}\text { Bias } \\
\times 10^{-7} \\
\quad(6)\end{array}$ & $\begin{array}{r}\text { Error } \\
\times 10^{-7} \\
(7)\end{array}$ & $\begin{array}{r}P 4 / P 0 \\
\times 10^{-7} \\
\quad(8)\end{array}$ & $\begin{array}{r}\text { bias } \\
\times 10^{-7} \\
(9)\end{array}$ & $\begin{array}{r}\text { Error } \\
\times 10^{-7} \\
(10)\end{array}$ & (11) & Error \\
\hline RXC J0003.8+0203 & 0.131 & 0.0041 & 0.031 & 0.220 & 0.1318 & 0.265 & 0.3395 & 0.058 & 0.213 & 0.0028 & 0.00079 \\
\hline RXC J0006.0-3443 & 0.448 & 0.0072 & 0.075 & 1.862 & 0.2348 & 1.055 & 0.2179 & 0.101 & 0.222 & 0.0190 & 0.00153 \\
\hline RXC J0020.7-2542 & 0.093 & 0.0033 & 0.025 & -0.094 & 0.1152 & 0.198 & 0.2389 & 0.050 & 0.215 & 0.0168 & 0.00116 \\
\hline RXC J0049.4-2931 & 0.091 & 0.0068 & 0.040 & 0.115 & 0.2156 & 0.477 & 1.0930 & 0.093 & 0.533 & 0.0026 & 0.00067 \\
\hline RXC J0145.0-5300 & 1.054 & 0.0057 & 0.113 & 1.000 & 0.1748 & 0.689 & 1.6230 & 0.078 & 0.556 & 0.0297 & 0.00170 \\
\hline RXC J0211.4-4017 & 0.307 & 0.0054 & 0.063 & -0.142 & 0.1871 & 0.213 & 1.3600 & 0.083 & 0.480 & 0.0041 & 0.00078 \\
\hline RXC J0225.1-2928 & 0.588 & 0.0146 & 0.121 & 4.883 & 0.4361 & 2.374 & 1.3040 & 0.239 & 0.759 & 0.0114 & 0.00139 \\
\hline RXC J0345.7-4112 & 0.152 & 0.0046 & 0.033 & 1.403 & 0.1572 & 0.743 & 0.8983 & 0.074 & 0.405 & 0.0044 & 0.00063 \\
\hline RXC J0547.6- & 0.081 & 0.0019 & 0.016 & 1.243 & 0.0543 & 0.348 & 0.7068 & 0.023 & 0.169 & 0.0129 & 0.00081 \\
\hline $\mathrm{RXCJ}$ & 0.064 & 0.0011 & 0.010 & 0.007 & 0.0298 & 0.056 & 0.0271 & 0.014 & 0.029 & 0.0057 & 0.00022 \\
\hline $\mathrm{RXC} \mathrm{JC}$ & 0.372 & 0.0063 & 0.084 & 6.137 & 0.1969 & 1.697 & 2.9580 & 0.088 & 0.742 & 0.0161 & 0.00143 \\
\hline RXC J0645.4-5413 & 0.258 & 0.0024 & 0.034 & -0.068 & 0.0720 & 0.095 & 0.1835 & 0.029 & 0.106 & 0.0121 & 0.00049 \\
\hline RXC J0821.8+0112 & 0.153 & 0.0188 & 0.083 & 6.717 & 0.5968 & 3.133 & 1.8220 & 0.303 & 1.075 & 0.0062 & 0.01416 \\
\hline RXC J0958.3-1103 & 0.100 & 0.0031 & 0.022 & 0.014 & 0.0922 & 0.195 & 0.1165 & 0.042 & 0.101 & 0.0029 & 0.00054 \\
\hline RXC J1044.5-0704 & 0.116 & 0.0008 & 0.011 & -0.013 & 0.0216 & 0.019 & -0.0029 & 0.009 & 0.019 & 0.0042 & 0.00024 \\
\hline RXC J1141.4-1216 & 0.041 & 0.0013 & 0.010 & 0.500 & 0.0399 & 0.198 & 0.0535 & 0.016 & 0.049 & 0.0052 & 0.00051 \\
\hline RXC J1236.7-3354 & 0.025 & 0.0051 & 0.018 & 0.145 & 0.1825 & 0.374 & 0.1157 & 0.081 & 0.244 & 0.0048 & 0.06762 \\
\hline RXC J1302.8-0230 & 0.722 & 0.0042 & 0.080 & 1.986 & 0.1345 & 0.647 & 0.8242 & 0.062 & 0.341 & 0.0215 & 0.02096 \\
\hline RXC J1311.4-0120 & 0.017 & 0.0004 & 0.003 & 0.024 & 0.0096 & 0.025 & 0.0066 & 0.004 & 0.010 & 0.0029 & 0.00026 \\
\hline RXC J1516.3+0005 & 0.075 & 0.0025 & 0.018 & 0.315 & 0.0710 & 0.235 & 0.2417 & 0.033 & 0.132 & 0.0059 & 0.00054 \\
\hline RXC J1516.5-0056 & 0.534 & 0.0065 & 0.076 & 8.604 & 0.2268 & 1.691 & 0.8404 & 0.091 & 0.394 & 0.0160 & 0.00135 \\
\hline RXC J2014.8-2430 & 0.026 & 0.0007 & 0.006 & 0.206 & 0.0203 & 0.091 & 0.0427 & 0.009 & 0.034 & 0.0053 & 0.00022 \\
\hline RXC J2023.0-2056 & 0.042 & 0.0118 & 0.035 & -0.199 & 0.4147 & 0.448 & 0.5655 & 0.178 & 0.594 & 0.0191 & 0.00130 \\
\hline RXC J2048.1-1750 & 0.760 & 0.0050 & 0.073 & 3.350 & 0.1311 & 0.813 & 1.8790 & 0.055 & 0.429 & 0.0460 & 0.00494 \\
\hline RXC J2129.8-5048 & 0.034 & 0.0059 & 0.052 & 2.631 & 0.2068 & 1.115 & -0.0427 & 0.096 & 0.160 & 0.0479 & 0.02450 \\
\hline RXC J2149.1-3041 & 0.005 & 0.0019 & 0.005 & 0.793 & 0.0538 & 0.262 & 0.0218 & 0.024 & 0.038 & 0.0038 & 0.00047 \\
\hline RXC J2157.4-0747 & 1.376 & 0.0240 & 0.251 & -0.691 & 0.7871 & 0.862 & 3.1270 & 0.392 & 1.709 & 0.0517 & 0.00343 \\
\hline RXC J2217.7-3543 & 0.039 & 0.0025 & 0.014 & 0.449 & 0.0658 & 0.237 & 0.1529 & 0.029 & 0.122 & 0.0019 & 0.00047 \\
\hline RXC J2218.6-3853 & 0.492 & 0.0016 & 0.035 & 0.351 & 0.0439 & 0.189 & 0.1159 & 0.021 & 0.063 & 0.0188 & 0.00057 \\
\hline RXC J2234.5-3744 & 0.101 & 0.0030 & 0.018 & -0.060 & 0.0900 & 0.129 & 0.2564 & 0.040 & 0.102 & 0.0136 & 0.00150 \\
\hline RXC J2319.6-7313 & 0.340 & 0.0047 & 0.058 & -0.119 & 0.1469 & 0.154 & 0.2151 & 0.065 & 0.178 & 0.0187 & 0.00103 \\
\hline
\end{tabular}

Notes. The cluster centers were not excised for the analysis results shown in this table. The power ratio parameters have been determined for an aperture with a radius of $R_{500}$. The corresponding results with center excision are given in Table 1 . For each of the power ratio parameters we provide the value of the noise contribution to the power ratio result (bias) which has been subtracted from the measured result to provide the value listed in Cols. 2, 5, and 8. The uncertainties determined from the Poissonisation simulations are listed in Cols. 4, 7, and 10 (error). The center shift statistic parameter $w$ and its uncertainty are listed in Cols. 11 and 12.

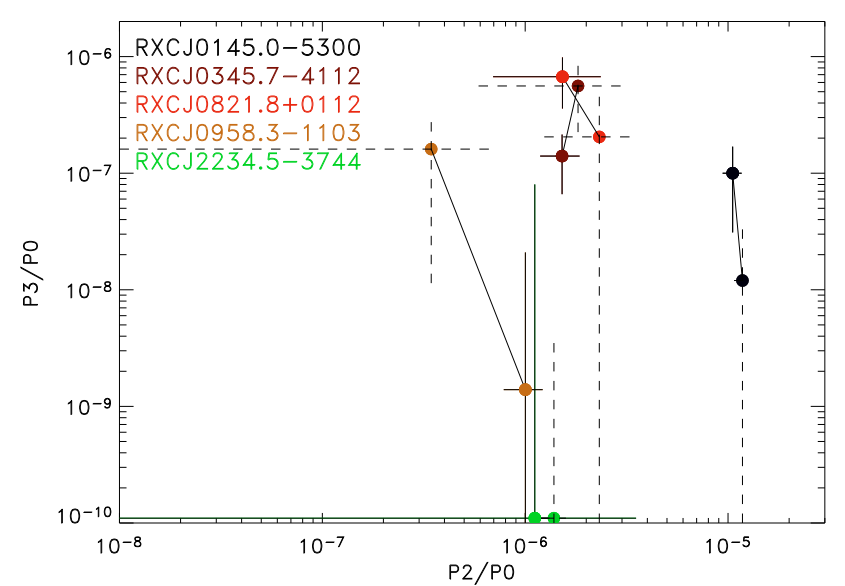

Fig. A.2. Comparison of the power ratio results $P 2 / P 0$ and $P 3 / P 0$ for five of the REXCESS clusters with two separate exposures. The results approximately agree within the combined errors, supporting our approach of estimating uncertainties with Poisson Monte Carlo simulations.

means their mean noise added structure parameters should be slightly biased high. However, the scatter is expected to give a good representation of the Poisson noise uncertainty of the observed data. We therefore use the standard deviation of these 200 Poisson simulations as a measure of the uncertainty for the power ratio parameters. Figure 1 shows the same data as Fig. A.1 but with the uncertainties determined from the Poissoniation simulations. The errors are considerably larger for the clusters with highly significant signals, but are similar near the significance threshold. Therefore our suspicion was correct that we need a different assessment for the measurement uncertainties than that given simply by the bias.

We can further check if the large uncertainties that we obtained are realistic. For this we compare the results of the analysis of five REXCESS clusters where we have multiple observations. These five clusters nicely cover a range of different morphologies. We show the results in Fig. A.2. The results for separate observations of the same cluster are different but all overlap with their uncertainties. The second observations have in some cases significantly lower exposure times, which increases the uncertainty. This test illustrates that the relatively large error estimates we calculate for the power ratios are well-justified.

We note once again that these uncertainties come from an end-to-end test of the analysis, since all the analysis steps of the power ratio determination are performed on the Poisson 


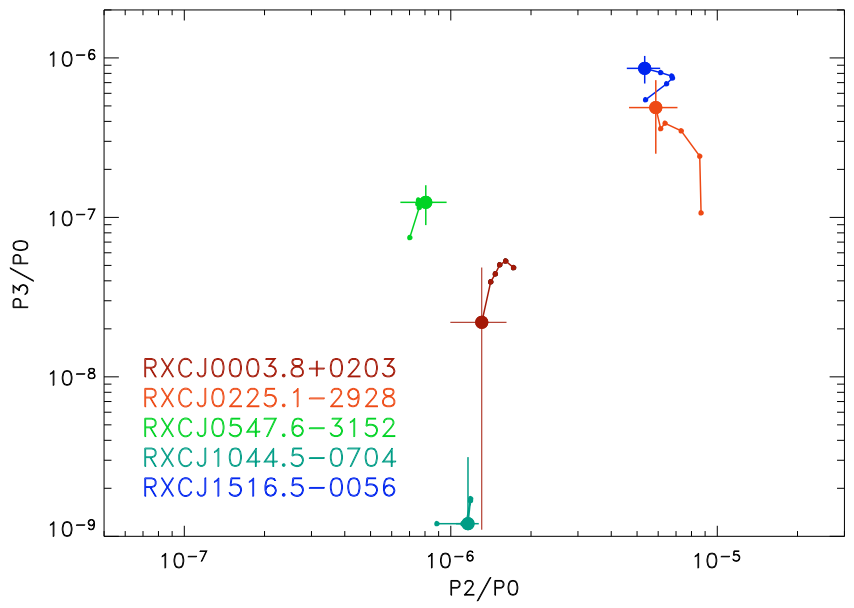

Fig. A.3. Influence of the sampling point spread function (PSF) of the $\mathrm{X}$-ray images on the derived power ratios. The heavy points give the unsmoothed result with XMM-Newton resolution. The smaller points connected by a line show the effect of successive smoothing by a Gaussian of width $4,8,15,30,60$ arcsec width. The error bars shown for the unsmoothed data points are those obtained from the Poissonisation simulations.

resampled images (i.e., including the centring and bias subtraction on top of the application of the power ratio formulae).

\section{A.2. Influence of the aperture radius and angular resolution on the power ratio results}

In the next test we explored the effect that the angular resolution of the observation has on the results. Using four clusters which again cover an interesting range of morphologies we determined the power ratios as a function of increased smoothing of the cluster images. The results are displayed in Fig. A.3. The change of the power ratios is shown as a function of successive smoothing by a Gaussian of width $4,8,15,30$, and 60 arcsec. For all four clusters the change is small, smaller than or roughly comparable to the typical errors of the overall measurement. Therefore the angular resolution of the observations is not an issue for the sample or for the comparison to other observations (e.g. clusters at higher redshifts observed at lower angular resolution).

To obtain an overview on the dependence of the power ratios on the aperture radius, we have calculated the power ratios for 10 different radii for all clusters starting with $0.1 \times R_{500}$ and increasing in steps of $0.1 \times R_{500}$. Due to the large powers of $R^{\prime}$ that appear in Eqs. (3) and (4), structure near the aperture radius is most heavily weighted. This became very obvious in the first tests we performed before removing point sources. Even only moderately strong point sources near $R_{\text {ap }}$ have a clear effect on the orientation of the multipoles and this effect decreases for smaller radii. Therefore we can expect that different structural features in the clusters become important for different values of $R_{\text {ap. }}$. We have clearly seen this in the visual inspection of the results. Figure A.4 shows the change of the power ratios with $R_{\text {ap }}$ for some examples of the REXCESS sample. Since we are mostly interested in global cluster parameters, e.g. in the study of the correlation of the structure parameters with other global parameters determined within $R_{500}$ (e.g. Pratt et al. 2007, 2009), we have concentrated on the results obtained for $R_{\text {ap }}=R_{500}$. Small changes in $R_{\text {ap }}$ leave the clusters in the same parameter range, while changes of the order of $0.5 R_{500}$ can give quite different results.

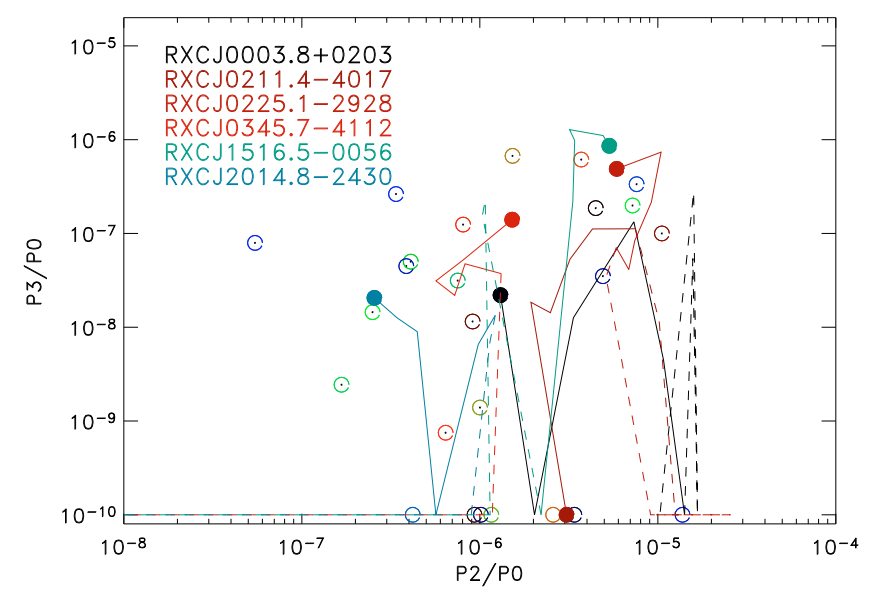

Fig. A.4. Effects of changing the aperture radius, $R_{\text {ap }}$, on the power ratios illustrated for six of the REXCESS clusters with different morphologies. The aperture radius is decreased from $R_{500}$ (marked as solid dot) to $0.5 R_{500}$ in steps of $0.1 R_{500}$ (shown as solid line) and further decreased to $0.1 R_{500}$ (shown as dashed line). The parameter distribution for the remaining REXCESS clusters is indicated by open symbols.

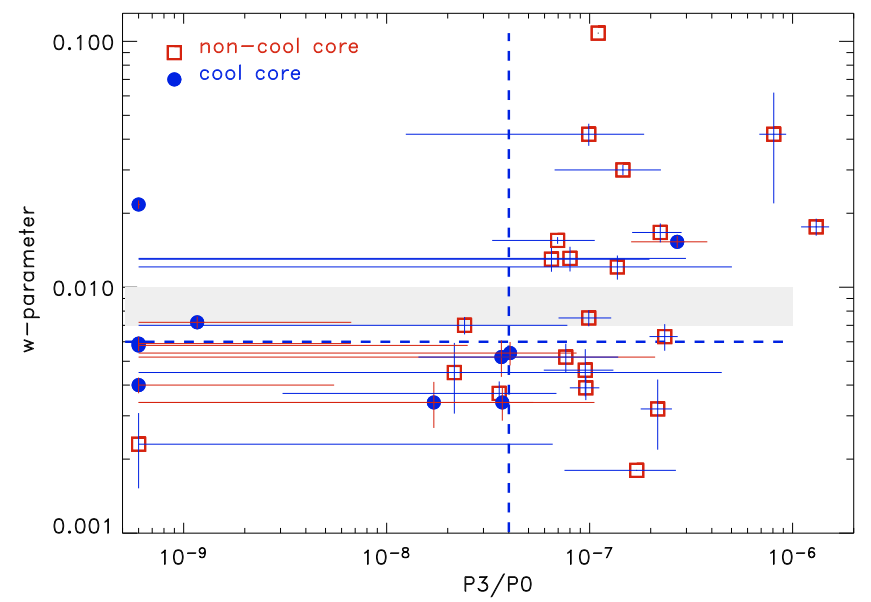

Fig. A.5. $w$ versus $P_{3} / P_{0}$ for the 31 clusters of the REXCESS sample. This figure is similar to Fig. 2, but now $P_{3} / P_{0}$ is determined for an aperture radius $R_{\text {ap }}=0.7 R_{500}$. The dashed blue lines and the grey bar are the same as in Fig. 2 for better comparison. The parameter values shown here were derived for the core excised images. We identify the clusters by their cool core properties as explained in the text.

\section{A.3. Comparison of power ratios and centre shifts}

In comparing the results of the power ratios with the centre shifts, we were anticipating that centre shift measures would be more sensitive to the central regions while power ratios are most sensitive to the outermost zones. To investigate this in more detail, we have looked at the correlation of $P_{3} / P_{0}$ with $w$, as shown in Fig. 2 for an aperture of $R_{500}$, but now as a function of the aperture radius. Table 3 gives the correlation coefficients for six aperture radii between $0.5 R_{500}$ and $R_{500}$. We clearly note a very sharp maximum of the correlation coefficient for an aperture radius between $0.7-0.8 R_{500}$. The distribution of the two substructure measures for an aperture radius $R_{\text {ap }}=0.7 R_{500}$ is shown in Fig. A.5. The points are now visually more correlated than in the corresponding Fig. 2. We also note a that cool cores are more clearly as being less morphologically disturbed; the classification of the power ratio parameter is now practically as tight as that of the $w$ parameter. The only cool core cluster that is classified by both methods as disturbed is RXC J1302.8-0230, which 
appears in the upper right quandrant. This system has a cool core that is clearly off-set from the large scale cluster center. These results thus seem to further encourage us to think about useful combinations of power ratio parameters from different radii to construct a more sensitive substructure measure.

\section{References}

Ameglio, S., Borgani, S., Pierpaoli, E., \& Dolag, K. 2007, MNRAS, 382, 397 Ameglio, S., Borgani, S., Pierpaoli, E., et al. 2009, MNRAS, 394,479 Arnaud, M., Pointecouteau, E., \& Pratt, G. W. 2007, A\&A, 474, 37

Arnaud, M., Pratt, G. W., Piffaretti, R., et al. 2009, A\&A, in press [arXiv: 0910.1234]

Beers, T. C., Gebhardt, K., Huchra, J. P., et al. 1992, ApJ, 400, 410

Bird, C. M., \& Beers, T. C. 1993, AJ, 105, 1586

Biviano, A., Murante, G., Borgani, S., et al. 2006, A\&A, 456, 23

Böhringer, H., Schuecker, P., Guzzo, L., et al. 2001, A\&A, 369, 826

Böhringer, H., Schuecker, P., Guzzo, L., et al. 2004, A\&A, 425, 367

Böhringer, H., Schuecker, P., Pratt, G. W., et al. 2007, A\&A, 469, 363

Böhringer, H. 2008, in The Universe in X-rays, ed. J. E. Trümper, \& G. Hasinger (Springer), 395

Borgani, S., Murante, G., Springel, V., et al. 2004, MNRAS, 348, 1078

Borgani, S., \& Kravtsov, A. 2009, Invited Review to appear on Advanced Science Letters (ASL), Special Issue on Computational Astrophysics, ed. L. Mayer [arXiv:0906.4370]

Bower, R. G., Benson, A. J., Malbon, R., et al. 2006, MNRAS, 370, 645

Buote, D. A. 2002, in Merging Processes in Cluster of Galaxies, ed. L. Feretti, I. M. Gioia, \& G. Giovannini (Dordtrecht: Kluwer), in press [arXiv: astro-ph/0106057]

Buote, D. A., \& Tsai, J. C. 1995, ApJ, 452, 522

Buote, D. A., \& Tsai, J. C. 1996, ApJ, 458, 27

Buote, D. A., \& Xu, G. 1997, MNRAS, 284, 439

Chen, Y., Reiprich, T. H., Böhringer, H., Ikebe, Y., \& Zhang, Y.-Y. 2007, A\&A, 466,805

Croston, J. H., Pratt, G. W., Böhringer, H., et al. 2008, A\&A, 487, 431

Croton, D. J., Springel, V. W., Simon D. M., et al. 2006, MNRAS, 365, 11

Dolag, K., Borgani, S., Murante, G., \& Springel, V. 2009, MNRAS, 399, 497

Evrard, A. E., Mohr, J. J., Fabricant, D. G., \& Geller, M. J. 1993, ApJ, 419, 9

Fabian, A. C., Crawford, C. S., Edge, A. C., \& Mushotzky, R. F. 1994, MNRAS, 267, 779

Fabjan, D., Borgani, S., Tornatore, L., et al. 2010, MNRAS, 401, 1670

Fitchett, M., \& Webster, R. 1987, ApJ, 317, 653

Girardi, M., Fadda, D., Escalera, E., et al. 1997, ApJ, 490, 56

Gomez, P. L., Pinkney, J., Burns, J. O., et al. 1997, ApJ, 474, 580

Guo, Q. 2009, Ph.D. Thesis, Ludwig-Maximilians Universität München

Haarsma, D. B., Leisman, L., Donahue, M., et al. 2010, ApJ, 713, 1037

Hashimoto, Y., Böhringer, H., Henry, J. P., Hasinger, G., \& Szokoly, G. 2007a, A\&A, 467, 485

Hashimoto, Y., Henry, J. P., Böhringer, H., \& Hasinger, G. 2007b, A\&A, 468, 25

Henry, J. P., Evrard, A. E., Hoekstra, H., Babul, A., \& Mahdavi, A. 2009, ApJ, 691, 1307

Isobe, T., Feigelson, E. D., \& Nelson, P. I. 1986, ApJ, 306, 490

Jeltema, T. E., Canizares, C. R., Bautz, M. W., \& Buote, D. A. 2005, ApJ, 624, 606

Jeltema, T. E., Hallman, E. J., Burns, J. O., \& Motl, P. M. 2008, ApJ, 681, 167
Kravtsov, A., Vikhlinin, A., \& Nagai, D. 2006, ApJ, 650, 128

Kay, S. T., da Silva, A. C., Aghanim, N., et al. 2007, MNRAS, 377, 317

Kolokotronis, V., Basilakos, S., Plionis, M., \& Georgantopoulos, I. 2001, MNRAS, 320, 39

Mantz, A., Allen, S. W., Rapetti, D., \& Ebeling, H. 2008, MNRAS, 387, 1179

Maughan, B. J., Jones, C., Forman, W., \& Van Speybroeck, L. 2008, ApJS, 174, 117

Mazzotta, P., Rasia, E., Moscardini, L., \& Tormen, G. 2004, MNRAS, 354, 10

Melott, A. L., Chambers, S. W., \& Miller, C. J. 2001, ApJ, 559, L75

McCarthy, I. Balogh, M. I., Babul, A., Poole, G. B., \& Horner, D. J. 2004, ApJ, 613,881

McCarthy, I., Babul, A., Bower, R. G., \& Balogh, M. I. 2008, MNRAS, 368, 1309

Mohr, J. J., Evrard, A. E., Fabricant, D. G., \& Geller, M. J. 1995, ApJ, 447, 8

Mohr, J. J., Fabricant, D. G., \& Geller, M. J. 1993, ApJ, 413, 492

O'Hara, T. B., Mohr, J. J., Bialek, J. J., \& Evrard, A. E. 2006, ApJ, 639, 64

O'Hara, T. B., Mohr, J. J., \& Sanderson, A. J. R. 2007, ApJ, submitted [arXiv: 0710.5782]

Pinkney, J., Roettiger, K., Burns, J. O., \& Bird, C. M. 1996, ApJS, 104, 1

Poole, G. B., Fardal, M. A., Babul, A., et al. 2006, MNRAS, 373, 881

Poole, G. B., Babul, A., McCarthy, I. G., et al. 2007, MNRAS, 380, 437

Poole, G. B., Babul, A., McCarthy, I. G., Sanderson, A. J. R., \& Fardal, M. A. 2008, MNRAS, 391, 1163

Pratt, G. W., Böhringer, H., Croston, J. H., et al. 2007, A\&A, 461, 71

Pratt, G. W., Croston, J. H., Arnaud, M., \& Böhringer, H. 2009, A\&A, 498, 361

Pratt, G. W., Arnaud, M., Piffaretti, R., et al. 2010, A\&A, 511, A85

Press, W. H., Teukolsky, S. A., Vetterling, W. T., \& Flannery, B. P. 1992, Numerical Recipes (Cambridge University Press)

Puchwein, E., Sijacki, D., \& Springel, V. 2008, ApJ, 687, L53

Rasia, E., Mazzotta, P., Borgani, S., et al. 2005, ApJ, 618, 1

Rasia, E., Ettori, S., Moscardini, L., et al. 2006, MNRAS, 369, 2013

Reiprich, T. H., \& Böhringer, H. 2002, ApJ, 567, 716

Richstone, D., Loeb, A., \& Turner, E. L. 1992, ApJ, 393, 477

Rosati, P., Borgani, S., Norman, C. 2002, ARA\&A, 40, 539

Rozo, E., Wechsler, R. H., Koester, B. P., et al. 2007, ApJ, submitted [arXiv: astro-ph/0703571]

Sanderson, A. J. R., Edge, A. C., \& Smith, G. P. 2009, MNRAS, 398, 1698

Sarazin, C. L. 1986, Rev. Mod. Phys., 58, 1

Schuecker, P., Böhringer, H., Reiprich, T. H., \& Feretti, L. 2001, A\&A, 378, 408

Schuecker, P., Böhringer, H., Collins, C. A., \& Guzzo, L. 2003a, A\&A, 398, 867

Schuecker, P., Caldwell, R. R., Böhringer, H., et al. 2003b, A\&A, 402, 53

Slezak, E., Durret, F., \& Gerbal, D. 1994, AJ, 108, 1996

Sijacki, D., Pfrommer, C., Springel, V., \& Enßlin, T. A. 2008, MNRAS, 387, 1441

Springel, V. 2005, MNRAS, 364, 1105

Springel, V., \& Hernquist, L. 2003, MNRAS, 339, 312

Springel, V., White, S. D. M., Jenkins, A., et al. 2005, Nature, 435, 629

Trümper, J. 1993, Science, 260, 1769

Valdarnini, R. 2006, New Astr., 12, 71

Valdarnini, R., Ghizzardi, S., \& Bonometto, S. 1999, New Astr., 4, 71

Ventimiglia, D. A., Voit, G. M., Donahue, M., \& Ameglio, S. 2008, ApJ, 685, 118

Vikhlinin, A., Voevodkin, A., Mullis, C. R., et al. 2003, ApJ, 590, 15

Vikhlinin, A., Kravtsov, A. V., Burenin, R. A., et al. 2009, ApJ, 692, 1060

Voit, M., Balogh, M. L., Bower, R. G., Lacey, C. G., \& Bryan, G. L. 2003, ApJ, 593, 272

Voit, G. M. 2005, Rev. Mod. Phys., 77, 207

Yang, H.-Y. K., Ricker, P. M., \& Sutter, P. M. 2008, ApJ, 699, 315 